\title{
CONTROLLING THE UNCONTROLLABLE: A NEW APPROACH TO DIGITAL STORYTELLING USING AUTONOMOUS VIRTUAL ACTORS AND ENVIRONMENTAL MANIPULATION
}

\author{
A Thesis \\ presented to \\ the Faculty of California Polytechnic State University, \\ San Luis Obispo
}

\author{
In Partial Fulfillment \\ of the Requirements for the Degree \\ Master of Science in Computer Science
}

by

Matthew James Colón

March 2010 


\section{(C) 2010 \\ Matthew James Colón \\ ALL RIGHTS RESERVED}




\section{COMMITTEE MEMBERSHIP}

TITLE:

CONTROLLING THE UNCONTROLLABLE: A NEW APPROACH TO DIGITAL STORYTELLING USING AUTONOMOUS VIRTUAL ACTORS AND ENVIRONMENTAL MANIPULATION

AUTHOR:

Matthew James Colón

DATE SUBMITTED:

March 2010

COMMITTE CHAIR: $\quad$ Dr. Franz Kurfess, Ph.D.

COMMITTEE MEMBER: $\quad$ Dr. Clark Turner, J.D., Ph.D.

COMMITTEE MEMBER: $\quad$ Dr. Zoë Wood, Ph.D. 


\begin{abstract}
CONTROLLING THE UNCONTROLLABLE: A NEW APPROACH TO DIGITAL
\end{abstract}

STORYTELLING USING AUTONOMOUS VIRTUAL ACTORS AND

ENVIRONMENTAL MANIPULATION

Matthew James Colón

In most video games today that focus on a single story, scripting languages are used for controlling the artificial intelligence of the virtual actors. While scripting is a great tool for reliably performing a story, it has many disadvantages; mainly, it is limited by only being able to respond to those situations that were explicitly declared, causing unreliable responses to unknown situations, and the believability of the virtual actor is hindered by possible conflicts between scripted actions and appropriate responses as perceived by the viewer. This paper presents a novel method of storytelling by

manipulating the environment, whether physically or the agent's perception of it, around the goals and behaviors of the virtual actor in order to advance the story rather than controlling the virtual actor explicitly. The virtual actor in this method is completely autonomous and the environment is manipulated by a story manager so that the virtual actor chooses to satisfy its goals in accordance with the direction of the story. Comparisons are made between scripting, traditional autonomy, Lionhead Studio's Black $\&$ White, Mateas and Stern's Façade, and autonomy with environmental manipulation in terms of design, performance, believability, and reusability.

It was concluded that molding an environment around a virtual actor with the help of a story manager gives the actor the ability to reliably perform both event-based stories while preserving the believability and reusability of the actor and environment. While autonomous actors have traditionally been used solely for emergent storytelling, this new storytelling method enables them to be used reliably and efficiently to tell event-based stories as well while reaping the benefits of their autonomous nature. In addition, the separation of the virtual actors from the environment and story manager in terms of design promotes a cleaner, reusable architecture that also allows for independent development and improvement. By modeling artificial intelligence design after Herbert Simon's "artifact," emphasizing the encapsulation of the inner mechanisms of virtual actors, the next era of digital storytelling can be driven by the design and development of reusable storytelling components and the interaction between the virtual actor and its environment.

Keywords: artificial intelligence, intelligent agent, storytelling, digital storytelling, scripting, autonomy, virtual actor, virtual environment, story manager, environmental manipulation 


\section{TABLE OF CONTENTS}

LIST OF TABLES ...................................................................................... vii

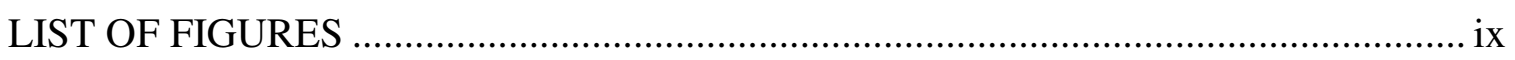

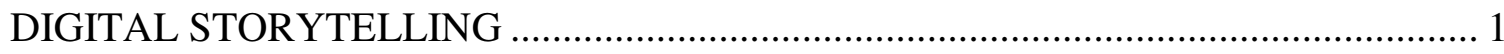

SURVEY OF STORYTELLING APPROACHES ................................................... 3

Event-based Storytelling and the Use of Scripting ............................................... 3

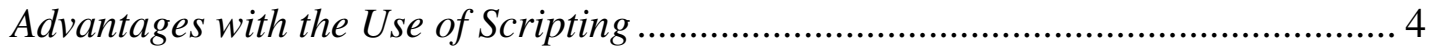

Disadvantages with the Use of Scripting ............................................................ 5

Emergent Storytelling and the Use of Autonomy ................................................. 7

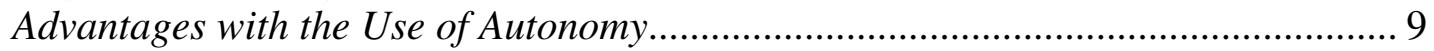

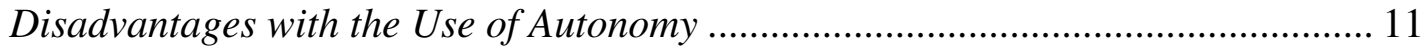

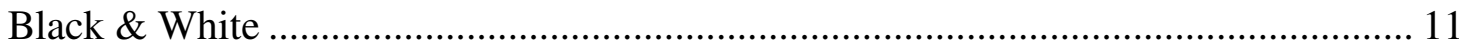

Advantages with Black \& White's Method of Storytelling ...................................... 13

Disadvantages with Black \& White's Method of Storytelling ................................ 15

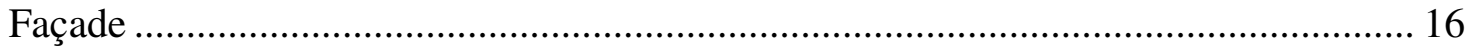

Advantages with Façade's Method of Storytelling ................................................. 18

Disadvantages with Façade's Method of Storytelling ............................................ 19

KEY ELEMENTS OF DIGITAL STORYTELLING METHODS .............................. 20

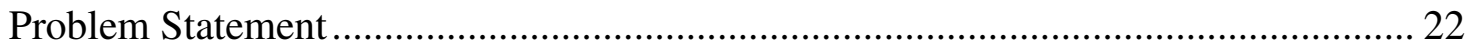

STORYTELLING WITH ENVIRONMENTAL MANIPULATION ............................ 22

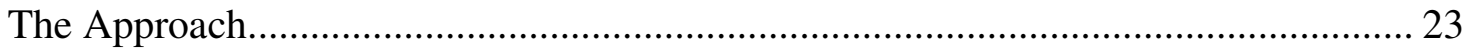

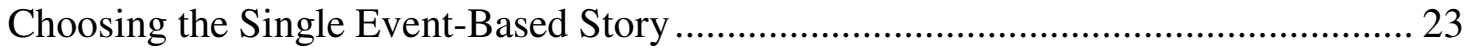

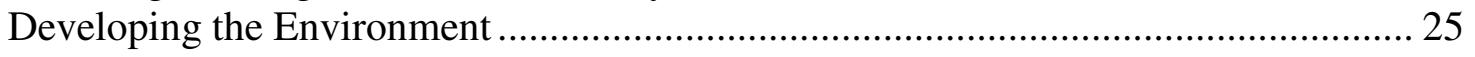

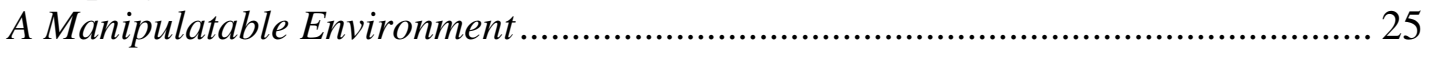

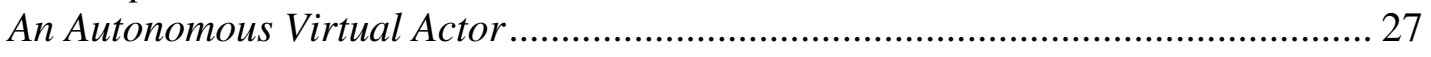

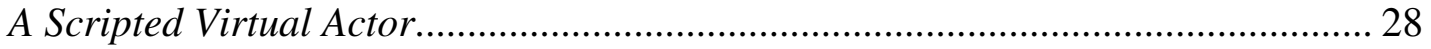

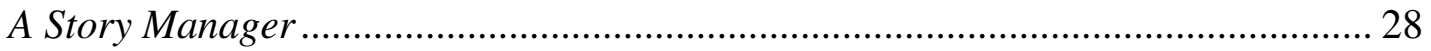

Comparing the Different Virtual Actors .............................................................. 29

Evaluating the Virtual Actors .......................................................................... 32

The Scripted Virtual Actor: Traditional Story ..................................................... 32

The Scripted Virtual Actor: Modified Story ........................................................... 32

The Autonomous Virtual Actor without Environmental Manipulation: Traditional

Story ....................................................................................................... 33

The Autonomous Virtual Actor without Environmental Manipulation: Modified

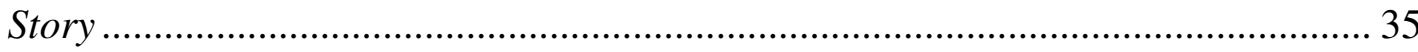


The Autonomous Virtual Actor with Environmental Manipulation: Traditional

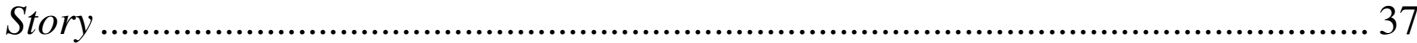

The Autonomous Virtual Actor with Environmental Manipulation: Modified

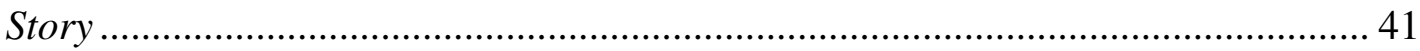

Using Environmental Manipulation for Multiple Event-Based Stories...................... 43

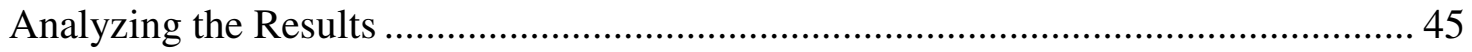

COMPARISONS WITH OTHER STORYTELLING APPROACHES ....................... 45

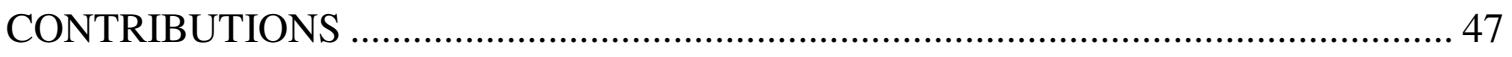

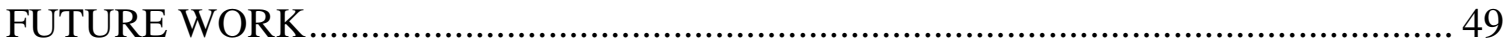

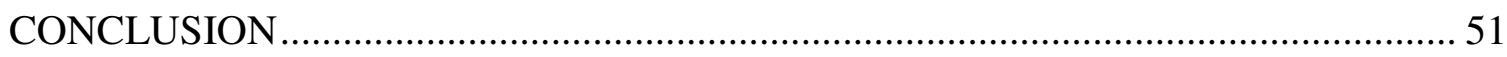

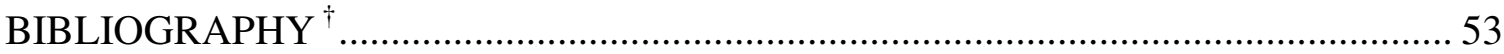




\section{LIST OF TABLES}

Table 1: The strengths and weaknesses of scripting, autonomy, Black \& White, and

Façade in terms of digital storytelling criteria.

Table 2: Results of tests $1-3$ for the scripted virtual actor performing the traditional story.....

Table 3: Results of tests $1-3$ for the scripted virtual actor performing the modified story

Table 4: Results of test 1 for the autonomous virtual actor without environmental manipulation performing the traditional story.

Table 5: Results of test 2 for the autonomous virtual actor without environmental manipulation performing the traditional story.

Table 6: Results of test 3 for the autonomous virtual actor without environmental manipulation performing the traditional story

Table 7: Results of test 1 for the autonomous virtual actor without environmental manipulation performing the modified story. 36

Table 8: Results of test 2 for the autonomous virtual actor without environmental manipulation performing the modified story.

Table 9: Results of test 3 for the autonomous virtual actor without environmental manipulation performing the modified story.

Table 10: Results of test 1 for the autonomous virtual actor with environmental manipulation performing the traditional story. 38

Table 11: Results of test 2 for the autonomous virtual actor with environmental manipulation performing the traditional story.

Table 12: Results of test 3 for the autonomous virtual actor with environmental manipulation performing the traditional story.

Table 13: Results of test 1 for the autonomous virtual actor with environmental manipulation performing the modified story.

Table 14: Results of test 2 for the autonomous virtual actor with environmental 
manipulation performing the modified story.

Table 15: Results of test 3 for the autonomous virtual actor with environmental manipulation performing the modified story.

Table 16: Results of test 1 for the autonomous virtual actor with environmental manipulation performing one of the multiple event-based stories. 44

Table 17: Results of test 2 for the autonomous virtual actor with environmental manipulation performing one of the multiple event-based stories.

Table 18: Results of test 3 for the autonomous virtual actor with environmental manipulation performing one of the multiple event-based stories.

Table 19: The strengths and weaknesses of environmental manipulation in terms of digital storytelling criteria compared with the other storytelling approaches..... 46 


\section{LIST OF FIGURES}

Figure 1: Screenshot of an unforeseen situation in Guild Wars. ..................................... 7

Figure 2: Order of story events for the two stories used in this exploration..................... 24

Figure 3: Graphical representations of the virtual actor and environment. .................... 27

Figure 4: Screenshot of the visual elements of the virtual actor and environment........... 28

Figure 5: Story event order in the traditional and modified stories. .............................. 31

Figure 6: Screenshot of virtual actor being influenced towards a story event................. 39 


\section{DIGITAL STORYTELLING}

Video game graphic engines are reaching incredible levels of realism, creating breathtaking virtual worlds and beautiful scenery. The maturity of the graphics field is allowing the field of artificial intelligence in video games to be explored and matured. In many cases today, games are being advertised for their achievements in artificial intelligence rather than graphics; F.E.A.R. (First Encounter Assault Recon), by Monolith Productions [F.E.A.R.], mentions its artificial intelligence more times on its box than it does its graphical effects. In addition, artificial intelligence in video games is becoming scrutinized more and more often by players. For example, a reoccurring discussion on the GWOnline forums [GWOnline] for Arena.net's Guild Wars [Guild Wars] is on the poor intelligence of the computercontrolled allies and how many players desire either improved artificial intelligence or more explicit control for the players over these allies.

Artificial intelligence plays an important role in digital storytelling, or the performance of a story using virtual actors in a virtual environment for an audience. A story consists of elements such as characters, locations, and events that are woven into a narrative within the story's fictional or non-fictional universe. The same universe can be visited in multiple stories, such as with the case of "fan fiction" in which a writer initially develops the universe in their story and then readers create their own stories within that same universe.

Just as human actors act out roles in plays, musicals, and movies, virtual actors governed by artificial intelligence perform their role in the digital world of the story being told. Sometimes this role is complex and intensively driven by the story, such as with the character, Alyx Vance from Half-Life 2 [Half-Life 2], who rescues the player early in the game and helps him extensively in the fight against a "Big Brother"-esque alien conqueror. Other times it is simple and peripheral to the story, such as with many of the residents of the towns in Bethesda's Elder Scrolls III: Morrowind [Morrowind], in which most actors simply wander around or stand in one location, giving simple responses when in dialogue with the player.

The digital story worlds that the virtual actors inhabit are referred to as the actor's environment. The environment consists of many different facets. One such facet is the "physical" representation of the 
story world, such as the scenery, terrain, and architecture of the world which is primarily for the viewing pleasure of the audience. Another is the implicit representation of environment properties which is interpreted by the actor. For example, an environment can specify that a diamond is valuable and a lion is dangerous regardless of their physical appearance, so a virtual actor playing the role of a thief can understand that it should collect diamonds because they are valuable and that it should run away from lions because they are dangerous.

Finally, while there are many other aspects of environmental design, the audience in particular is important for storytelling with virtual actors. The audience can be one who merely views the story being told or can be an active participant in the story, usually referred to as a "player." A digital character controlled by the audience is also considered part of the environment to the virtual actor because the virtual actor may interact with the human-controlled character or be affected by the character's actions. The audience may be allowed to affect the progress of the story, such as by talking to certain virtual actors or making changes in the virtual environment. However, on the opposite end of the spectrum, the audience can be restricted from interfering and simply observe the telling of the story.

Digital storytelling has matured much from its beginnings to its current state. Early games required very little artificial intelligence (if it is even considered that) in order to progress the story being told. For example, in Nintendo's Super Mario Bros [SMB], the most guidance that the player received in terms of what to do next in the story is from several humanoid mushrooms who tell the player, "Thank you Mario! But our princess is in another castle!" simply meaning the game is not over yet. However, digital storytelling is becoming vital for story progression in modern games. Valve's Half-Life 2 did an amazing job with storytelling by subtly guiding the player along the story. For example, near the beginning of the game the player is in an apartment complex which is raided by police soon after his arrival. The player's exit is cut off by police officers, and as the player is running through the apartment complex, other residents are telling him to get to the roof, which is stressed further by police coming up every staircase and thus cutting off more possible exits. The player was not simply left to wander around the apartment complex before discovering what to do next. Instead, artificial intelligence was creatively used to instill a sense of urgency; with police chasing from behind and cutting off every downward exit as well as residents frantically trying to get themselves and the player to safety, the player quickly realizes that he has only one 
hope: getting to the roof and as quickly as possible.

A unique characteristic of digital storytelling is that these stories are remotely and dynamically performed. This means that these stories are performed without human supervision at the time that the story is being told and they are performed "live" for the audience. Books and movies are remotely read and watched out of the presence of the story's writer, but are statically "performed" because they have been printed to a static medium that will not change each time the story is read or watched. Oppositely, plays and musicals are dynamically performed in front of a live audience, but either the story's writer or a director of some sort is on hand locally to help the performance if anything would go awry, such as by giving a forgetful actor their lines or informing them that they missed their cue. Digital stories, on the other hand, are performed both without any human guidance on hand (a video game studio cannot afford to ship an employee with each copy of their game) and are performed dynamically for their audience since the story's performance is initiated at its audience's command. Because of this, great care is needed in developing a strong digital storytelling approach to ensure that the intended story is correctly performed each time despite the remote and dynamic performance.

\section{SURVEY OF STORYTELLING APPROACHES}

\section{Event-based Storytelling and the Use of Scripting}

Because most video games are telling a story in which specific events must occur at certain times, one method of designing artificial intelligence is through scripting, or the creation of artificial intelligence through the use of a scripting language. This method gives most of the control to the story's director by explicitly stating how a virtual actor should act in each known possible situation, exhibiting a top-down approach like that of directing a movie; just as a movie director gives each human actor a movie script, the story's director can give each virtual actor artificial intelligence from a high-level scripting language [Barnes]. These AI scripts allow for the virtual actor to respond to interactive cues that are set up in the environment and act out their AI according to their script, such as when the audience moves to a certain location or talks to a certain actor. In addition, this method of storytelling many times uses "cut scenes," or scenes in which the audience's interaction is limited so that virtual actors follow their script exactly in order 
to advance the story [Barnes]. For example, the real-time strategy game, Warcraft III by Blizzard Entertainment, Inc. [Warcraft], has cut scenes before and after the player completes a particular mission in which virtual actors are used to set up and conclude the mission's story without letting the player intervene.

An example of event-based storytelling in written literature is the Choose Your Own Adventure series of children's books, published by Bantam Books from 1979 to 1998 [CYOA]. During the telling of the story in these books, the reader is prompted with several actions or events to choose from and a page number to jump to for each choice. Depending on what choices are made, the story unfolds and concludes in different ways, some better than others; one path through the story may lead to the main character's death while another leads the main character to happiness and prosperity. In terms of the literary actors, or the characters in the story, they "perform" only what has been explicitly written by the story's writer to happen depending on each choice the reader makes. The choices and consequences have been already designed by the story's writer, and the reader experiences the story in the way (or ways) that the writer meant for it to be told.

\section{Advantages with the Use of Scripting}

In terms of storytelling, scripting is advantageous for event-based storytelling because of its reliability and its ability to set the mood or scene for the story. First of all, because the actors are given explicit instructions on how to behave in every situation that they will encounter (or at least the ones that are relevant), their behavior is reliable. Because of this, a single event-based story or multiple event-based stories can be performed exactly as the developers designed it to. When a video game is shipped to the consumers, the developers can be sure that the artificial intelligence exhibited on each consumer's computer will be what they had intended because the virtual actors have no other unknown choices in behavior. In addition, scripting can be used to reliably set a mood or scene for the story. Half-Life 2 does these in many ways, one example being the scene when the player enters an apartment building as mentioned before. Another such use of scripting for setting the mood is when the player is in a territory that is inhabited by creatures known as antlions. If the player sets foot on the sandy beach in this area, these antlions emerge from the sand and relentlessly assault the player. Since the player must navigate across this extensive beach in order to progress, this simple scripted behavior of making the antlions attack if the player steps 
onto the sand instills a sense of fear into the player, causing them to carefully build ad hoc bridges across the sandy terrain in fear of the consequences of a mistake.

\section{Disadvantages with the Use of Scripting}

While scripting proves advantageous in many situations, it has several problems with reusability, replay value, unscripted situations, believability, and audience interaction. Because each particular situation (or type of situation) must be scripted in order for the virtual actor to behave correctly according to the director, there is a severe limit on the amount of actions the actor can perform - only as many as have been scripted. Scripting thus presents several problems. First of all, much of the design of the artificial intelligence has to be customized for each new game, making reusability of scripted actors difficult. For

example, if a scripted actor was designed explicitly for a first-person shooter game to be a soldier proficient with machine guns, that same actor could not be directly imported into a medieval role-playing game because of the obvious lack of automatic weaponry. In addition, Orkin mentions that a problem in implementing scripting is that the designers can get "bogged down scripting the actions of individual characters" [Orkin]. Because individual characters need to be uniquely scripted in many cases, reusability can be difficult even within the same game, let alone different games.

Second of all, the story may have less replay value because the virtual actor's behavior can become too predictable. Paul Tozour, of Ion Storm Austin, remarks on the predictability of scripted actors:

Every gamer has had the experience of playing through a game and finding that he can read the gameplay like a script. 'When I try to open the door to Mr. Giotti's room, one of the bad guys on the other side of the door says, 'It's Max Wayne! Nothing can stop that guy!' And the other bad guy says, 'Oh yeah? Well how about this?' and shoots a missile at the door and blows it up, conveniently allowing me, Max Wayne, to step through...

Scripted AI can also greatly reduce the game's replayability. It can pervert the core gameplay into a form of memorization. The first time through the dungeon, you get slaughtered, but load your last saved game, and you have the advantage of knowing exactly where the enemies are and how they will attack you. [Tozour]

In addition, believability of the virtual actor is hindered by both encounters with unscripted situations and when scripted scenes conflict with what the virtual actor "should" be doing in the particular situation. Jonty Barnes, of Lionhead Studios, comments on stories that allow the player to drive the story: 
Games featuring dynamic physical simulation and artificial intelligence (AI) introduce the possibility of undefined circumstances occurring, and can afford the player, rather than the game designers, the freedom to set the agenda. Is it possible to add a storyline to such open-ended gaming worlds without jeopardizing the player's suspension of disbelief? [Barnes]

Game designers must take great care to cover all expected scenarios that a virtual actor may encounter; if one actor misses his cue, it may cause the story to come to a halt. For example, in the game, Torchlight, a virtual actor named Brink is meant to accompany the player down into the depths of the mines beneath the city of Torchlight. At one point in the mines, a cut-scene begins where the player encounters a monster that casts a spell on Brink, causing him to mutate into a monster as well and then attack the player. However, if Brink gets left behind in the mines due to the player not taking care to keep him close, the player may encounter this monster alone and because Brink is not around to give the monster its cue, the cut-scene begins, but never progresses, showing the player and monster staring at each other. The only way to continue the story is for the player to skip the cut-scene [Torchlight].

In an example of scripted scenes conflicting with believable behavior, Guild Wars uses cinematic cut-scenes regularly to progress the story, but sometimes the scripted artificial intelligence that governs the virtual actors' behaviors does not take into account the actions of autonomous creatures around them.

Figure 1 shows one such example: 


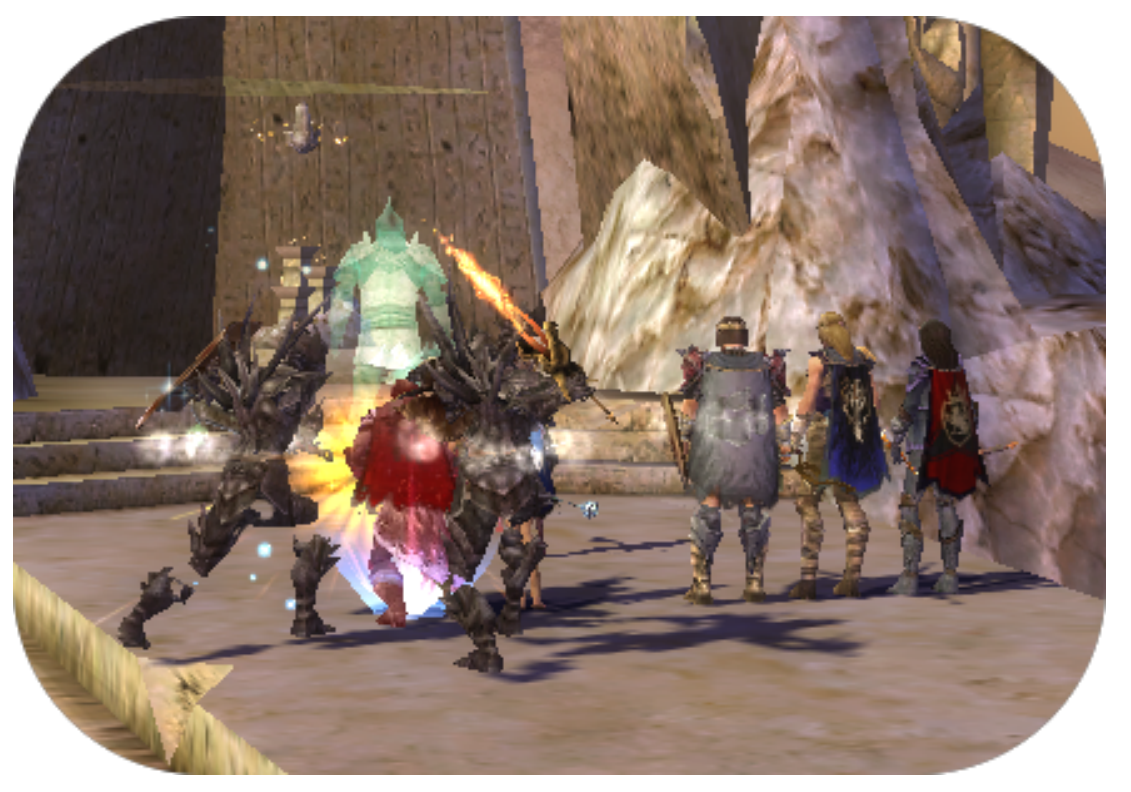

Figure 1: Screenshot of an unforeseen situation in Guild Wars.

A story event in Guild Wars in which the players' virtual actors are listening to instructions from the teal, translucent Ghostly Hero. However, all virtual actors are ignoring the attacking enemy creatures, making the behavior of the actors seem much less believable.

In these situations, the believability of the virtual actors is hindered by their obviously unintelligent behavior. If the actors were aware of the situation, they could fight back, run, or do something about the unexpected situation. However, because the actor is not acting on its own freewill but on the explicit direction of the story's director, their behavior may be contradictory to the situation, thus unintelligent behavior may arise such as in the above figure.

Finally, because the storytelling performed by scripted actors mirrors the vision developed by the story's director, the audience may not be allowed at times to interact with the virtual actors in order to prevent unknown situations from occurring. While the reasoning is obvious - a story may not be very believable or able to be completed if the audience kills a main character or throws themselves off of a cliff - it limits the immersion and influence of the audience in the story. No longer are they an active participant, but just an observer of somebody else's story.

\section{Emergent Storytelling and the Use of Autonomy}

In many cases, a story does not need to be limited by specific storytelling events, but instead can be influenced in seemingly any direction. This method is referred to as emergent storytelling. Emergent 
storytelling can be used when the director of the story does not want to force a single path to be followed or conclusion to be reached. In essence, the story "emerges" from the interactions between the virtual actors and the environment. This method of storytelling primarily uses a bottom-up approach, focusing on the development of the characters and environment of the story so that they can respond to the many different interactions and choices that the actor will encounter. Relating back to the Choose Your Own Adventure stories, these stories would be truly emergent if the reader could make their own choices in each situation and the literary actors could intelligently respond to those choices.

In terms of interactive emergent storytelling with virtual actors, the same paradigm is followed; the director allows the audience and environment to aid or interfere in the telling of a story, and the virtual actors respond accordingly to the different story progressions. "Emergent gameplay is born when players have the freedom to explore and solve problems in any way they choose, and the game world responds appropriately" [Orkin]. Marc Cavazza, Fred Charles, and Steven J. Mead state that in emergent stories, "[the emphasis is] on the actor's behavior rather than on explicit plot representation or narrative control," but some accommodation may be desired for "authoring aspects of the baseline narrative" [Cavazza]. This emphasis allows for the development of believable virtual actors that can perform a story based on their environment and their interactions with it. While the focus of emergent storytelling is on providing opportunities to guide the progression of a story, constraints may be placed on the types of situations that emerge as to not stray from the genre of the story. For example, if an emergent story is based on a love story, the situations that emerge should not allow the audience to kill the couple.

Emergent storytelling makes use of autonomous actors - actors that have their own goals and subgoals that govern their behavior - to tell the story. Jeff Orkin, of Monolith Productions, states that "autonomous characters can handle unexpected events that a script might not have anticipated, producing emergent gameplay" [Orkin]. Because the entire purpose of the actor is to satisfy its own goals with some amount of randomization, different stories are created through the satisfaction of the goals in different ways.

An example of emergent storytelling in video games can be seen in the Nintendo DS game, Scribblenauts. The primary focus of the game is for the player to collect objects known as "starites" in order to complete each level. This is achieved by entering words for objects that the player would like to 
use in the level, such as "bear," "plane," or "Cthulhu." However, the way that the player combines these objects to obtain the starites us left up to their imagination. For example, the player finds that a starite is located high up on a tree. They could create a ladder to climb up and grab the starite, create a football and kick it to hit the starite out of the tree, or create a beaver to cut down the tree. While the goal is clearly presented, it is up to the player to creatively define the journey [Scribblenauts].

\section{Advantages with the Use of Autonomy}

The use of autonomous virtual actors has the advantages of creating unique stories each performance, intelligently responding to any situation in the actor's environment, believability, reusing virtual actors, and allowing the audience to be a part of the storytelling. As stated above, because of the random nature of the internal mechanisms of an autonomous virtual actor, new and different stories can be developed each time the story is performed. The "desires" of the virtual actor will be somewhat different each performance, meaning that the virtual actor will make different choices and its behavior will be affected by those choices. This leads to a multitude of stories than can be told within the same environment with the same virtual actors, increasing the replayability of the story. Orkin states that "the tremendous success of Grand Theft Auto 3's [GTA3] open-ended 'sandbox' style game design is a testament to the game players' satisfaction with emergent gameplay [Orkin]."

Autonomous characters are built to intelligently respond to the environment that they are in without a need for prior environmental knowledge. By having the autonomous actor choose its behaviors in real-time, they are not limited like scripted actors are in terms of intelligently behaving in unexpected situations. This is because autonomy uses a bottom-up approach to determine behaviors from interactions with the actor's environment instead of using scripting's top-down approach to determine behaviors from specific situations. For example, Orkin mentions one player's experience with autonomy in Monolith's No One Lives Forever 2: A Spy in H.A.R.M.'s Way (NOLF2):

Players of NOLF2 recounted stories on Web forums about emergent gameplay they experienced. $N O L F 2$ follows a linear story, but each level gives players freedom to explore and choose how to overcome obstacles. Players take on the role of international super-spy Cate Archer. The game starts in Japan where Cate meets a contact, Yamata-san, who gives her a first mission. Cate needs to infiltrate a village guarded by fierce ninjas and photograph a secret meeting. Yamata-san is armed for self-defense, in the unlikely scenario that Cate lures a ninja all the way down to the start 
of the level. A NOLF2 player on the Web posted a clever approach to overcoming the difficulty of fighting the ninjas in the village. The player used Cate's tazer to knock out Yamata-san and carry him into the village to an area with lots of enemies. Upon waking, Yamata-san disposes of the ninjas far more deftly than the player could have at the start of the game. The player used the game mechanics of the tazer and carrying bodies, and was rewarded by Yamata-san reacting appropriately by fighting the ninjas that surrounded him when he woke up. Yamata-san handled the situation autonomously, without a script defining how to react to the unanticipated situation of waking up from a tazer shock in a hostile environment. A minor character in the game design took a major role in this player's experience due to the player's decisions. [Orkin]

This example also shows the ability to reuse virtual actors because of their ability to adapt to whatever environment they are placed into. The first environment for Yamata-san was peacefully away from danger because he and the player had to discuss the objectives of the mission. However, without requiring any changes to the internal mechanisms of the actor himself, Yamata-san was able to adapt to a hostile environment in which he must fight for his life. This reusability of autonomous actors has many benefits, such as being able to spend development time on the adaptability of virtual actors to their environments rather than specifically programming the behaviors for each actor.

In addition, the ability of the autonomous virtual actors to make their own decisions based on their own free will gives the actor more believability. Instead of the audience witnessing purely the actions of the actor, they can see the decision-making process of the actor as well, if implemented. Facial expressions, body language, and idle behavior can be used to reflect the internal state of the actor's decision-making process. For example, let's assume the audience decides to attack a peasant in full view of a soldier. Instead of having the soldier immediately respond by attacking the audience, in which the audience is left to determine why the soldier is attacking, the witnessing character could express shock (witnessing that an innocent character is under attack), then anger (determining the source of the attack), then perform their behavior.

Finally, because autonomous virtual actors are able to intelligently adapt to unknown situations in their environments, the audience is able to influence the actors much more than when scripting is being used. Not only do the interactions between the audience and the actors increase replayability, as mentioned before, the actors are able to intelligently react to the audience because the audience is part of its environment. Instead of story directors needing to take precautions against the audience causing actors to get into unknown states, as with the use of scripting, they can use the adaptability of autonomous actors to 
allow the audience to have an active participant role in the telling of the story.

\section{Disadvantages with the Use of Autonomy}

The freedom of the autonomous virtual actors to make their own decisions and behave according to their own free will introduces several problems with their use in storytelling, namely their unreliable nature and their inability to perform an event-based story. Because they ultimately are based on some amount of randomness, a story director cannot assume that the autonomous actor will perform certain behaviors at certain times. Each performance of the story is different, even if ever so slightly, thus each audience's experience cannot be known ahead of time. This means that if a story director had a particular direction he wanted the story to go, such as he wanted two virtual actors to fall in love within the story, he could not reliably use autonomous actors to perform that story; while one audience may witness the director's desired story being performed, another may see the two actors not care for each other or never even meet. This is seen even further through attempting to perform an event-based story. If, for example, a story director wanted to use autonomous actors to perform a single story that involved ten story events that must be performed in a certain order, there is one correct performance out of 3628800 possible

performances and thus a $2.76 \times 10^{-5} \%$ chance of the autonomous actor correctly performing that story, assuming that each story event can only be performed once. If events can be performed multiple times, the chance of an autonomous actor correctly performing the single story is reduced to zero, making it infeasible to tell a single story while allowing the complete freewill of the autonomous actors.

\section{Black \& White}

One method of combining the power of scripting and autonomy for storytelling is seen in Lionhead Studio's Black \& White [B\&W], a video game in which the player's character is a god trying to win the worship of the population in a polytheistic world and who has a large, autonomous creature as his physical representation in the world. The game is structured to be a large playground for the player if desired, allowing the player to have explicit and implicit control over his creature and the autonomous actors in the game, and each situation the player encounters can be responded to in a number of ways. Barnes recognizes, though, that by giving the player such open-ended control and the ability to sway the 
desires, goals, and behaviors of both the autonomous creature and the autonomous villagers, the player maintains control of the telling of the story instead of the director and could easily cause unforeseen situations to arise. However, if scripting was extensively used to control the telling of the story, the player's influence would be severely limited and possibly detract from the player's reception of the story. As mentioned before, Barnes poses the question, "is it possible to add a storyline to such open-ended gaming worlds without jeopardizing the player's suspension of disbelief?" [Barnes]

Black \& White reconciles this issue through the use of scripted and autonomous actors for storytelling, giving most of the storytelling control to the audience and virtual actors while maintaining a general direction for the story. This method is a combination of scripting's top-down approach and autonomy's bottom-up approach for storytelling in which most of the story progression is done by the audience and autonomous virtual actors, but the director explicitly advances the main story at key intervals through the use of scripted sequences, known as Challenges. A Challenge could be such things as entertaining events, tutorial exercises, puzzles, or events that advance the telling of the story. For example, Barnes discusses one such Challenge:

One of the first Challenges written was the "Lost Brother." This Challenge begins with a woman emerging from her house in prayer for the safety of her ill brother who has become lost in the woods. Informed of her plight, the players are free to do what they choose. They can ignore the prayers of the woman completely. They can reunite the grateful siblings. They can find and kill the lost brother, and taunt the grieving woman by holding his lifeless body just out of her reach. They can take the woman to the forest where her brother lays, wait until the pair is happily walking back to the woman's house, and then kill them both maliciously and without warning. [Barnes]

Challenges were designed to be initiated by the player, but not require them to complete the Challenge immediately. For example, one Challenge requires the player to strike awe into a villager unimpressed with the player's divinity. In order to win the worship of this unbelieving villager, one option is to come back to the villager once the player's creature has grown significantly in size, which requires several hours of gameplay to achieve. In the meantime, the player can complete other Challenges, explore the land, or train his creature, allowing him to place the Challenge of impressing the unbelieving villager aside until it can be completed. However, allowing Challenges to be put aside poses the problem of creating undesirable circumstances. For example, in the "Lost Brother" Challenge, if the player sets aside 
the Challenge, his creature could decide to eat the brother while the player is somewhere else in the land. This could cause a scripted cinematic to begin about how the player maliciously completed the Challenge while the player had no role in its completion. Special care was taken to remedy this problem, such as restricting the creature from interacting with actors or objects under the control of a script without the explicit instruction of the player.

\section{Advantages with Black \& White's Method of Storytelling}

Black \& White's method of storytelling is able to achieve the general story desired by the director while allowing the audience to personalize the flow of the story, as well as combining the advantages of scripting and autonomy. Despite the fact that autonomous virtual actors are used, the director is able to tell the general story throughout the game by using scripting only when necessary and only when the player desires. This allows the player to set the agenda, progressing the story in a personalized manner. Instead of restricting the player's ability to complete a Challenge to only a few ways, the scene is simply prepared for the player, then given over to him to do what he desires. As mentioned above, the "Lost Brother" Challenge can be completed in many different ways, limited only by the imagination - whether benevolent or malevolent - of the player.

This method also combines the advantages of scripting and autonomy. The story can easily be advanced through the scripted Challenges by explicitly controlling the behaviors of relevant virtual actors, and those actors can be used to set the scene for the player. For example, the sister in the "Lost Brother" Challenge emerges from her home, and kneels before the player, bowing down in prayer and despair, thus setting the mood of her situation through her speech and actions. In addition, her brother is seen sick and coughing, alone and lost in the forest, further showing the despair of the situation.

The advantages of autonomy are seen as well through the actions of the villagers and the player's creature. The villagers have a life primarily of working at different tasks to sustain the village, but are able to respond to changes in their environment and the interactions of the player. For example, villagers respond when they witness demonstrations of the player's power, such as when the player throws a fireball safely over a town. In addition, if the player picks up a large rock somewhere in the land and places it in the middle of the village, the villagers will become curious of their new monument, and eventually spend 
their evenings dancing around it in praise of the player's divinity, imbuing the rock with the power of their worship. This rock can then be used to win the worship of other villages by placing it in their town.

A comprehensive demonstration of the villagers' autonomy was seen in an attempt that was made by the author to win over a village that believed in a different deity. The player's direct influence can only extend a short distance from believing villages, so the author attempted to gain the worship of a distant village by carefully throwing a rock imbued with the worship of his village into the center of the remote village. When the rock was thrown, it rolled slowly into the center of the village. The witnessing villagers exclaimed their amazement at the incoming rock and the power that must have caused the rock to move into their domain. However, before the rock came completely to a stop, its final revolution caused it to crash into the village's town center. The once-awestruck villagers screamed and ran at the sight, their amazement turning to fear.

This event demonstrated the ability of the autonomous villagers to react to their environment through their interpretations of the rock and its source. First of all, it was recognized by the villagers as an uncommon event - large rocks usually do not tend to wander into town on their own - which caused them to stop their current actions and marvel at the sight. Second of all, they recognized that great power was needed to cause this event, which provoked their initial response of wonder. Finally, when the rock damaged their village, they responded in fear because the perceived purpose of this rock and its source changed from amazement to destruction, causing them to run for their lives. Not only does this example show the ability of the autonomous actors to respond to their environment, but also that their actions make them seem more believable. These villagers were able to react to an environment that was not familiar, yet responding in a way that humans would believe was intelligent behavior.

The player's autonomous creature also uses the advantages of autonomy through learning and through facial expressions and body language. The creature is initially a clean slate of autonomy; it knows only to do what it desires. For example, if it is hungry, it searches its environment for something that it believes will satisfy its hunger: wheat, fish, villagers, rocks, etc. It then learns from its actions through both the consequences of its actions and the feedback from the player, such as if the creature eats rocks and gets sick or eats a villager and gets disciplined by the player. From then on, the creature keeps in mind what it has learned when choosing what it wants to do. In addition, the creature uses facial expressions and body 
language to communicate to the player. For example, the creature is still unfamiliar with the world early in the game and looks frightened on occasion, showing worry on its face and pulling its arms close to it. Also, if the player does a miracle near the creature, it may look at what the player has done with wonder, pointing to what it saw (sometimes even trying to mimic what the player had done). These visual cues show the player that the creature is able to intelligently interpret and respond to its environment, visually mirroring aspects of the creatures internal processing so that the player can understand the thoughts and actions of the creature.

\section{Disadvantages with Black \& White's Method of Storytelling}

While Black \& White limits the use of scripting primarily during Challenges, the scripted sequences may become repetitive when replayed and also cause believability issues when partnered with the unconstrained nature of the game. In terms of repetitiveness, the Challenges may become stale when replayed several times. For example, the tutorial for the game that shows the player how to use the controls and interact with his creature takes around half an hour to complete. Once the player has played through the game, he is familiar with such things as camera controls, village management, and creature interaction, and thus does not need to complete the scripted tutorial a second time. In addition, some Challenges may have solutions that are much more efficient and simpler than others. For example, one simple way to complete the "Lost Brother" Challenge if the player wants to be benevolent is to pick up the brother and place him next to his sister, thus completing the Challenge much faster than if he healed the brother or took the sister to the brother and in both cases waited for them to return. Once the player has experimented and found the simplest way to complete a Challenge, replaying it is not a challenge anymore but is simply a repetition of actions that were already learned.

Because explicit scripted events must occur within a world under the control of the player, there are times in which the believability of the sequence can be hindered by the player's actions. For example, as mentioned before, the opening sequence for the "Lost Brother" Challenge involves the sister pleading to the player to save her brother, and then the camera moves to show the sick brother lost in the forest.

However, if the player chooses to throw a fireball towards the forest and then quickly initiate the Challenge, the scripted sequence would begin, but the fireball now presents an unexpected threat to the 
brother. The story director can attempt to handle this situation several ways, all of which are not beneficial for the believability of the Challenge. One way is to remove the fireball from the world when the Challenge has begun to simply avoid having to handle an unexpected situation, but this negates the player's power to have control over their world. Another way is to allow the fireball to hit the forest, burning down the trees and killing the brother during the cinematic, but now the Challenge has been completed before it had even begun. The approach that was implemented in Black \& White was to allow the fireball to hit, but to grant invulnerability to scripted actors during Challenge cinematics. However, this means that the player watching the cinematic sees the sick brother in the middle of a burning forest, oblivious to his surroundings, who is then killed as soon as the cinematic ends. This combination of scripted scenes within a world controlled by the player presents many of these "Catch 22 " scenarios to the story director; no matter how these types of situations are handled, maintaining the believability of the scene becomes difficult.

\section{Façade}

Virtual actors developed with an emphasis on believability are able to provide a unique digital storytelling experience. Michael Mateas and Andrew Stern state the following:

Over the past decade, there has been a fair amount of research on believable agents - autonomous characters exhibiting rich personalities, emotions, and social interactions. Researchers have done comparatively little work, however, on how to integrate believable agents' reactive behavior with a story plot's more deliberative nature. Such integration would enable interactive, dramatic worlds. [Mateas]

Most work in this area is focused on extending emergent storytelling to allow for a drama manager, or computer-controlled story director, to pull a virtual actor towards a key story point rather than directly control him. In other words, a virtual actor behaves autonomously until the drama manager tells him he has to do something. The virtual actor responds, does his required task, and returns to autonomy, similar to in Black \& White except that the drama manager dictates when the story should progress rather than the player. More work needs to be done, though, in improving the virtual actor's ability to compromise between their own internal goals and the commands from the drama manager in order to prevent unbelievable behavior [Magerko]. In addition, the drama manager can be improved to analyze the 
emerging story that the player is creating and prevent the player from pursuing a story direction that cannot return to the general story [Magerko].

Façade, an interactive drama program developed by Michael Mateas and Andrew Stern, uses this approach to perform a general story while allowing the audience to influence its progression. The plot of Façade is the following:

You, the player, using your own name and gender, play the character of a longtime friend of Grace and Trip, an attractive and materially successful couple in their early thirties. During an evening get-together at their apartment that quickly turns ugly, you become entangled in the high-conflict dissolution of Grace and Trip's marriage. No one is safe as the accusations fly, sides are taken and irreversible decisions are forced to be made. By the end of this intense one-act play you will have changed the course of Grace and Trip's lives - motivating you to re-play the drama to find out how your interaction could make things turn out differently the next time. [Façade]

Combining autonomous virtual actors, a drama manager to help progress the story, and the ability for the player to interact with the actors and the environment, Façade tells the story about the evening get-together with Trip and Grace, but ultimately allows the player to guide its final destination.

Façade allows the audience to interact with the virtual actors and their environment physically and through natural language processing, and the drama manager guides the story based on the audience's influence. For example, the audience can pick up objects within the apartment, such as the phone or Trip's Magic 8 Ball, and incorporate that into the conversation. In addition, the audience can choose to comfort, hug, or kiss either Trip or Grace, eliciting a response appropriate for the story at that point; if kissed when he answers the door, Trip will remark that the audience is doing that "European thing," while if kissed during a heated argument later in the story, Trip will not know how to respond to the audience's unexpected advances. Finally, Trip and Grace can be interacted with through natural language, in which the audience can type in short sentences to speak to the couple. For example, at one point Trip asks the audience to look at a picture of Italy that he took during the couple's vacation. Specifically, he asks what one word comes to the audience's mind when looking at the picture. If the audience responds with "romance," Trip is happy because that was the word he was thinking of as well. If the audience responds with "Italy," he is a little disappointed, agreeing that it was indeed a picture of Italy, and encourages the audience to come up with another word. However, if the audience responds with unrelated words, Trip becomes discouraged trying to get the audience to pick the word he is thinking of. 
Depending on how the audience is influencing the story, the drama manager will choose the next story event to perform. For example, there is a scene where Trip wants to make an alcoholic drink for the audience. If the audience agrees, Trip becomes happy and goes to his mini-bar to whip up the drink.

However, if the audience says that they are not thirsty or only wants water, Trip becomes discouraged and eventually gives up the idea of showing off his bartending skills, taking the story in a different direction. The progression of story events is ultimately realized at the conclusion of the story in which Trip and Grace decide to stay together or leave each other. In addition, depending on whom the audience interacted with the most during the story, either Trip or Grace will be the person who initiates the final scene, reflecting on the importance of what the audience has told them about their relationship during the story. As a side note, the audience's influence may cause the drama manager to conclude the story before the climax. There are several ways that this can occur, primarily if the audience continually insults the couple or makes explicit attempts to break the couple up, such as by excessively hugging and kissing Grace in Trip's presence.

\section{Advantages with Façade's Method of Storytelling}

Façade's method of storytelling is advantageous in that it is able to perform a general story while allowing for the audience to influence its progression and the use of autonomous virtual actors maintains a high level of believability during the performance. The overall story about an evening get-together with Trip and Grace is maintained throughout the performance, yet the events that occur during the story are based on how the audience chooses to interact with the couple, leading to their marriage strengthening or disintegrating. In addition, the drama manager makes sure that the audience does not steer the story away from the general framework desired by the story's director. Either the drama manager will move towards a new story event, such as if the audience continually refuses Trip's offer to make them a drink, or the couple will decide that its time that the audience leaves in the case of inappropriate behavior.

The autonomous nature of Trip and Grace is essential for believability during the story. Especially when the audience is allowed to interact with the couple through natural language processing, believability is difficult to maintain if the virtual actors are not able to respond intelligently to the audience's interaction. However, the virtual actors respond to the audience with facial expressions and body language in addition to verbal responses. These subtle visual responses help the audience to know more about how the virtual 
actor is feeling in a given situation and in response to the audience's interaction. For example, Trip or Grace may tell the player that everything is fine in their marriage, but their expressions give away the underlying trouble. Also, when Trip and Grace are arguing about their marriage, if the audience chooses to tell the couple, "I love Grace," and proceeds to kiss her, both Trip and Grace become shocked and exchange awkward glances. All of this expresses the feelings of the virtual actor without speaking verbally to the audience.

\section{Disadvantages with Façade's Method of Storytelling}

While Façade demonstrates great progress in digital storytelling, there are several drawbacks to its approach, primarily restrictions with audience interaction, repetitive and predictable story events, unknown circumstances due to scripting, and believability issues with natural language interaction. First of all, while the audience is allowed to influence the outcome of the story, Façade does limit the amount of influence the audience can use. In comparison with the extensive amount of control given to the player in Black $\&$ White, Façade will only allow the audience to slightly sway the story. By using this system in which a drama manager guides the story from one event to another, a fully emergent story would require the knowledge of all the possible story events that the audience would be allowed to generate in order to use scripting to guide the story in that direction.

As mentioned before, the use of scripting can lead to stories that become repetitive and predictable over time and cannot handle unknown circumstances when they arrive. The drama manager does choose a few different story events during the performance in order to make each story somewhat different, but once the audience understands the best way to interact with the couple in order to influence the story in a particular way, predictability comes into play once again as it did with Black \& White's Challenges. For example, in the previously mentioned situation in which Trip asks the audience to think of one word that his picture represents, once the audience has seen that situation once, they know that "romance" is the word Trip is looking for. Also, Grace wants to hear from the audience that the apartment does need to be rearranged in order to spruce things up, and once the audience realizes this, they know to say that it does in order to be on good terms with Grace. In addition, because scripting is involved, situations may arise albeit rare - in which the virtual actors do not know how to respond. For example, when performing the 
final sequence in which Grace is leaving Trip, the author chose to hug and kiss Trip multiple times in an attempt to stop the couple from departing by any means necessary. Afterwards, Trip and Grace would no longer respond to the author's actions and words, yet would not complete that sequence of the story, permanently caught in a situation that their artificial intelligence knew not how to handle.

While not completely the fault of the artificial intelligence, natural language in Façade sometimes caused reactions from Trip and Grace that were not believable. For example, certain phrases used by the audience which are normally not provocative could cause the couple to become shocked at what the audience had said, looking quickly from the audience to each other in horror and confusion of how to respond. In addition, the meaning of the audience's message may be lost or distorted when it is interpreted by the artificial intelligence. During the heated arguments between the couple about the state of their marriage, the messages, "Trip, Grace loves you,” or, “Trip, you love Grace,” are misinterpreted as a statement that Trip might not love Grace despite the lack of a word with a negative connotation in the phrase. This makes it difficult for the audience to play the role of an impromptu marriage counselor if what they want the couple to understand can be misinterpreted by the natural language component of Façade.

\section{KEY ELEMENTS OF DIGITAL STORYTELLING METHODS}

The primary areas of strengths and weaknesses in these digital storytelling methods revolve around the design and reusability of the virtual actors, their ability to perform the desired story, their believability, and the amount of audience interaction allowed. First of all, design is important for virtual actors and their environments. Not only is implementation and maintenance of the virtual actor much easier with better design, a well designed actor and its environment need minimal modification to be reused in different scenarios. This translates to less work when creating new stories and characters as well as benefits in terms of being able to spend additional time upfront creating virtual actors since the work put into them will not be wasted.

Depending on the type of story being told, virtual actors must be used that can correctly and effectively perform the desired story. If a story should only follow one particular path, the virtual actors need to be able to stay on that path and play their appropriate roles throughout the story. Likewise, if the 
story is to be emergent, the virtual actors need to be able to respond to whatever situations that arise in their environment.

The believability of the virtual actors is vital to the audience's interpretation of the story. Just as movie actors must convince movie goers that they truly embody their character, virtual actors must exhibit the behavior of living, breathing characters rather than sequences of instructions. The more the virtual actor makes the audience forget they are watching a story or playing a video game, the more effective the story is when it is interpreted.

Finally, audience participation within a story helps the audience personalize their experience. By allowing the audience some or all control to dictate how the story will be performed, it increases the replayability of the story by encouraging the audience to change what happens each time the story is performed. When the audience sees themselves as a participant rather than an observer, the story becomes more alive.

Table 1 shows how well the digital storytelling methods discussed achieve these five criteria:

\begin{tabular}{|c|c|c|c|c|}
\hline & Scripting & Autonomy & Black \& White & Façade \\
\hline Design / Reusability & POOR & STRONG & UNKNOWN & UNKNOWN \\
\hline Event-Based Story Performance & STRONG & POOR & MEDIUM & STRONG \\
\hline Emergent Story Performance & POOR & STRONG & STRONG & MEDIUM \\
\hline Believability & MEDIUM & STRONG & MEDIUM & MEDIUM \\
\hline Audience Interaction & POOR & STRONG & STRONG & MEDIUM \\
\hline
\end{tabular}

Table 1: The strengths and weaknesses of scripting, autonomy, Black \& White, and Façade in terms of digital storytelling criteria.

As shown in Table 1, no single storytelling method is able to perform strongly in all categories. Scripting's main strength is that an event-based story can be performed, yet it lacks in the other aspects of storytelling. Autonomy shines in its ability to perform emergent stories believably while allowing the audience to participate and can also be reused because of its modular design, but is infeasible to use for event-based 
storytelling because of its random nature. Black \& White combines event-based and emergent storytelling, but because the focus is on the player controlling the progression of the story rather than the director, it is more that the player advances to a new point in the story rather than the story progressing in a timely manner. In addition, scripting introduces situations in which believability can be strained. Finally, Façade does well in performing an event-based story with minor emergence, but has issues with audience interaction in terms of natural language processing and the amount of control given to the audience. Also, while the virtual actors are made to be believable, natural language processing and scripting produce situations in which the audience's suspension of disbelief may be broken.

\section{Problem Statement}

An observation about the digital storytelling approaches above is that combining scripting and autonomy improves upon their weaknesses, but never overcomes them. This raises an important question: can a digital storytelling approach correctly perform event-based stories without relying on scripting so that it can perform strongly in terms of believability and reusability? Such a method is presented in this paper, which can be achieved through environmental manipulation.

\section{STORYTELLING WITH ENVIRONMENTAL MANIPULATION}

This paper presents a novel solution for storytelling which can reliably perform event-based and emergent stories through the use of autonomous actors. This approach proposes that instead of forcing an actor to follow the story, such as what is done with scripting and traditional story directors, let the actor choose to follow the story by making the choice to perform the next step of the story seem like the best choice for the actor to make. This can be done by molding the environment around the actor so that the satisfaction of its goals and the progression of the story are aligned. This can be done explicitly, such as by placing interactive objects or establishing barricades within the environment, or implicitly, such as by modifying the perception of objects in the environment and thus influencing one of the factors that the virtual actor uses to make its decisions. For example, if an uncontrolled, autonomous Goldilocks was placed in the Bears' house, she might decide to sit down in a chair first, then go to sleep, then eat some porridge, not following the traditional fairy tale at all. However, if a story manager manipulates the 
environment to make the bowl of hot porridge look very appetizing, she should choose to satisfy her hunger goals before her comfort goals despite her general "desire" for comfort being greater than hunger. From there, the environment can continue to be manipulated by making the cold porridge look appetizing, then the "just right" porridge, and so on in order to tell the traditional story. This approach verges on the issue of digital free will verses predetermination; the virtual actor has free will to determine what it wants to do, but the choices that it makes are influenced by the story manager towards a specific goal. This allows an autonomous virtual actor to be able to perform event-based stories correctly just as a scripted actor could, but by using autonomous virtual actors, a manipulatable environment, and a story manager in which all three are completely modular, the storytelling approach can be enhanced to perform strongly in all categories in Table 1.

\section{The Approach}

In order to demonstrate the capabilities of storytelling with environmental manipulation, it will be first shown that this approach can perform the simplest case: a single event-based story. The storytelling approach should demonstrate that a virtual actor is able to perform each event in the story in the necessary order while exhibiting aspects of believability in its behavior. After this, it will be shown that this approach can perform a modified version of the previous single event-based story. This will demonstrate the reusable nature of the approach in that none of the components' internal processing needs to be rewritten in order to work with the modified story's new event order. Finally, it will be shown that this approach can perform multiple event-based stories in which a choice at the beginning of the story determines which storyline is pursued. While this thesis will not demonstrate the approach performing emergent stories or allowing for audience interaction, this will be discussed as future work.

\section{Choosing the Single Event-Based Story}

Two different stories were used in order to analyze the reusability, correct story performance, and believability of a scripted actor, an autonomous actor without environmental manipulation, and the same autonomous actor with environmental manipulation. By using two different stories, criteria could be used to evaluate how well the virtual actors adhered to each story while not being modified from their original 
state. The two stories are based on the fairy tale, Goldilocks and the Three Bears, the first according to the traditional story (i.e. Goldilocks eats the Bears' porridge, sits on their chairs, and sleeps in their beds, going from Papa Bear's items to Mama Bear's and finally to Baby Bear's in each category) and the second rearranging the story events into a new version (i.e. Goldilocks eats Papa Bear's porridge, sits in his chair, and sleeps in his bed, then does the same for Mama Bear's items and then for Baby Bear's). Goldilocks and the Three Bears was chosen as the story universe because it is a familiar fairy tale in which people easily identify the important events of the story: the eating of each of the bears' porridge, the use of each of the bears' chairs, and the use of each of the bears' beds. Because it is familiar, those viewing this demonstration could easily determine when the story is not correctly being performed.

The stories consist of the following events in the order they must be completed:

Goldilocks and the Three Bears Traditional Version:

1. Eat Papa Bear's Porridge

2. Eat Mama Bear's Porridge

3. Eat Baby Bear's Porridge

4. Sit in Papa Bear's Chair

5. Sit in Mama Bear's Chair

6. Sit in Baby Bear's Chair

7. Sleep in Papa Bear's Bed

8. Sleep in Mama Bear's Bed

9. Sleep in Baby Bear's Bed

Goldilocks and the Three Bears Modified Version:

1. Eat Papa Bear's Porridge

2. Sit in Papa Bear's Chair

3. Sleep in Papa Bear's Bed

4. Eat Mama Bear's Porridge

5. Sit in Mama Bear's Chair

6. Sleep in Mama Bear's Bed

7. Eat Baby Bear's Porridge

8. Sit in Baby Bear's Chair

9. Sleep in Baby Bear's Bed

Figure 2: Order of story events for the two stories used in this exploration. 


\section{Developing the Environment}

In order to begin this exploration using autonomous virtual actors for performing a single story, several components related to the virtual actors and the environment needed to be developed:

\section{A Manipulatable Environment}

An environment needed to be created in which certain aspects could be modifiable. This environment would allow for an actor to evaluate the environment, move around in the environment, and know what locations are able to satisfy goals. When the environment is modified, it would change an autonomous actor's perception of the satisfiability of goal-satisfying locations, thus causing them to reevaluate any decision that it had made.

The environment that was created consists of square tiles in a two-dimensional grid. Each tile has $\mathrm{x}$ and $\mathrm{y}$ coordinates in the grid as well as references to non-diagonal adjacent tiles. This allows for the actor to use a simple searching algorithm to build a search tree and find the desired location to move to. In addition, goal-satisfying locations are recognizable as places in which the actor's goals can be satisfied and they have both a goal satisfaction type and perceived satisfaction value. This value is labeled "perceived" because each goal-satisfying location satisfies a goal by a fixed amount despite how satisfying the actor believes it is. When a goal-satisfying location is used in this environment, it is considered unusable for the remainder of the story. This was chosen because the stories used for this demonstration only require a location to be used once in the story, thus once all goal-satisfying locations are used, the story is finished. While the environment itself is simplistic, it contains the components needed to demonstrate the capabilities of environmental manipulation, primarily the ability to have the perceived satisfiability of the goal-satisfying nodes manipulated and thus alter the actor's perception of its environment.

Using Goldilocks and the Three Bears as the story model, the environment needed to have the nine locations of interaction in it as mentioned above. To achieve this, the two-dimensional tile-based environment was given nine goal-satisfying locations with the appropriate goal that it satisfies and a value representing the perceived satisfaction of the location, beginning at the actual satisfaction value. Those 
goal-satisfying locations as well as the graphical representation of the environment and virtual actor from the perspectives of the actor and the audience can be seen in Figure 3:

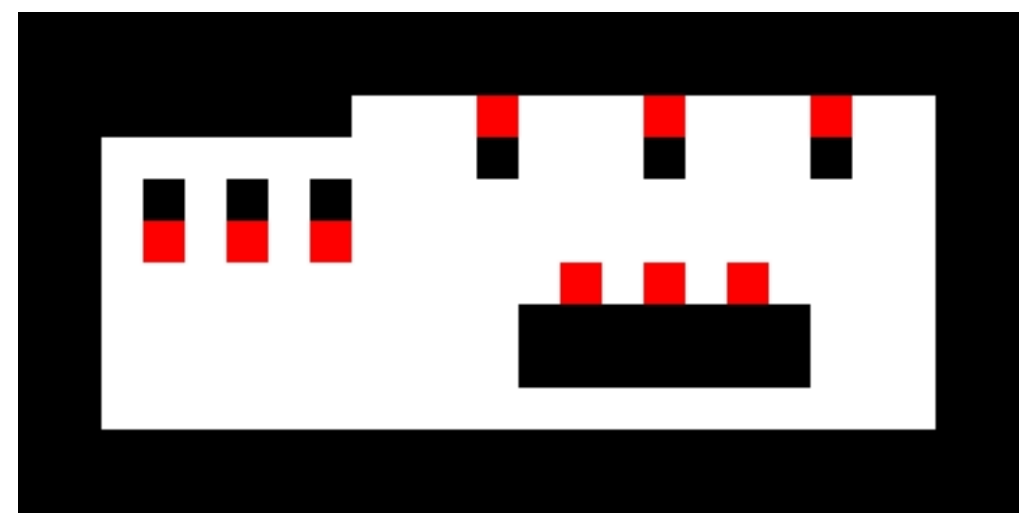

(a)

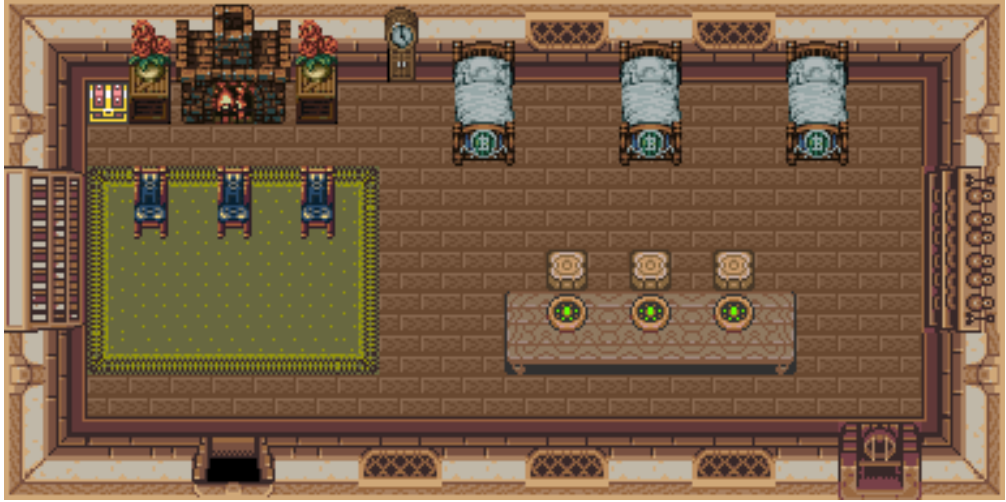

(b)

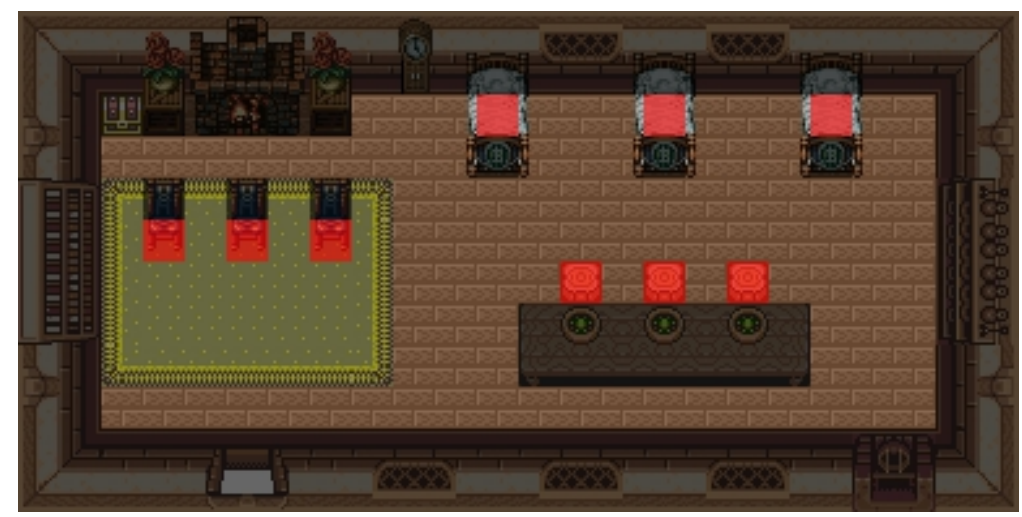

(c) 


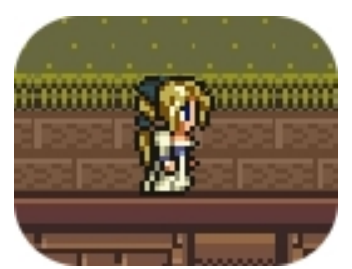

(d)

Figure 3: Graphical representations of the virtual actor and environment.

(a) The model representation of the environment from the perspective of the virtual actors, where white tiles represent open space, black tiles represent impassible tiles, and red tiles represent goalsatisfying locations.

(b) The graphical representation of the environment from the perspective of the viewer watching the story.

(c) The model representation overlaid on the graphical representation.

(d) The graphical representation of the virtual actor from the perspective of the viewer watching the story.

(Images used for the environment from Square's Final Fantasy VI [FFVI] and Nintendo's The

Legend of Zelda: A Link to the Past [Link], and images used for the virtual actor from Squaresoft's Final Fantasy VI [FFVI])

As a side note, the audience is not able to interact with the virtual actors in this demonstration because Goldilocks and the Three Bears revolves around Goldilocks' solitary exploration of the Bears' house, thus another entity in the story would not make sense. The effects of audience interaction with the storytelling approach will be discussed later.

\section{An Autonomous Virtual Actor}

An autonomous virtual actor would dynamically respond to its environment, making decisions on what actions to take depending on where it believes its goals could best be satisfied in the environment.

This autonomous actor has three goals: the satisfaction of hunger, of a need for comfort, and of a need to sleep. For each of these, the actor has a "desire" for how important it is to satisfy the particular goal. This "desire" increases by a random amount as the story progresses.

The actor initially looks through the environment in order to locate and remember all of the goalsatisfying locations at the start of the story. This can be done once at the beginning because the Bears' house will remain static throughout the story. If a particular story involves a dynamic environment, the actor could be designed to update its knowledge of the environment periodically. Additionally, the actor makes a decision to move to a location in the environment that satisfies a particular goal if the "desire" and the perceived satisfiability of that location combined is greater than any other combination of the two and is 
above some threshold. If no combination meets these requirements, the actor will idly move about the environment. In the case of multiple locations having the same highest satisfiability value, one is randomly chosen of that set. When a decision is made, the autonomous actor will then go to the goal-satisfying location, satisfy its goal, and then evaluate its environment to see where it desires to go next. If the environment changes immediately after a decision is made, the actor reassesses the satisfiability of the environment and makes a new decision.

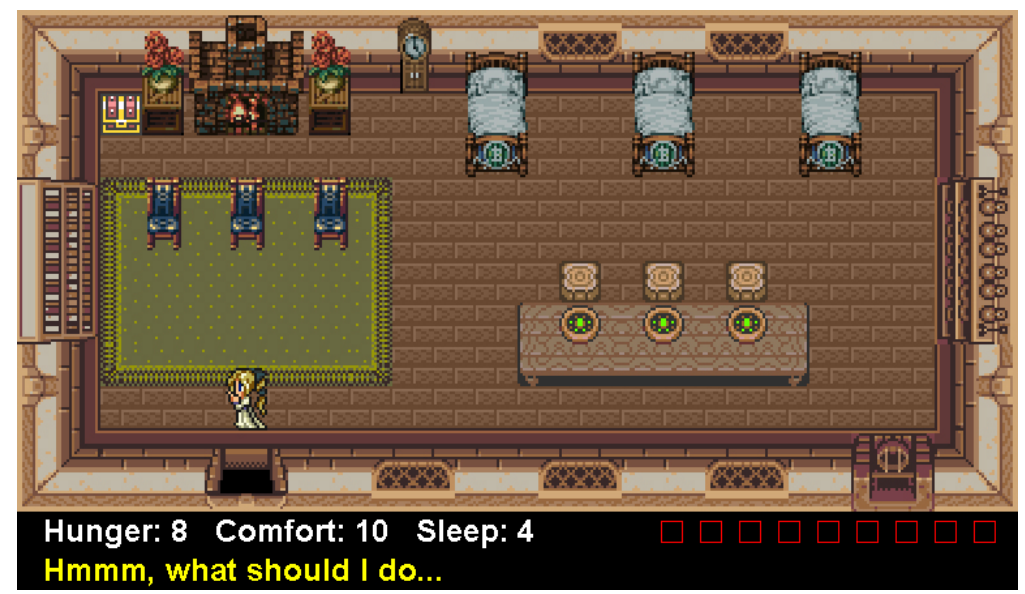

Figure 4: Screenshot of the visual elements of the virtual actor and environment. The depiction of the autonomous virtual actor and environment at the beginning of a story. Hunger, comfort, and sleep values, as seen in white text, slowly increase over time. In addition, the current decision (or indecision) of the actor is displayed in yellow text. Finally, the red squares represent whether a story event has occurred, being empty before and solid after the event was performed. A correct performance of the story would fill in each square from left to right in order.

\section{A Scripted Virtual Actor}

In order to compare the results between scripting and this new storytelling method, a scripted virtual actor needed to be developed. This scripted actor is given the locations to move to perform the events of the Goldilocks and the Three Bears story and their traditional and modified story order, thus acting according to the story's "script." When the story begins, the actor checks which location it should move to, moves to that particular location, and then repeats the process until it finishes its script.

\section{A Story Manager}

A story manager is used to observe the autonomous virtual actor and modify the perceived 
satisfaction values of goal-satisfying locations as needed. When the autonomous actor makes a decision, the story manager compares the chosen action with the next action required by the story. If the actions are different, the story manager improves the perceived satisfiability of the location that the story needs the actor to interact with and reduces the perceived satisfiability of the location chosen by the virtual actor. This is repeated every time the actor makes a decision that deviates from the intended story so that the location needed by the story seems to be able to grant the actor greater and greater satisfaction than any other location despite what the actor "desires" most at the time. Because the story manager increases the perceived satisfiability of the location that is currently needed by the story and decreases the rest of the locations every time the actor chooses to perform an action counter to the story, the actor will always eventually choose to perform the needed story event; it may just take some time. Sometimes the actor already wants to satisfy its goal in accordance to the story, so all that is necessary is to guide the actor to the particular location required by the story for that goal.

\section{Comparing the Different Virtual Actors}

In order to compare design, reusability, performance, and believability, the criteria that were used for an objective evaluation of the different actors consisted of the following:

Number of times the actor had to reevaluate its decision: Was the actor able to make a final decision after a reasonable amount of reevaluations? This is related to the performance and believability criteria mentioned before. If the actor can make a final decision within a reasonable number of reevaluations, it can be seen that the actor can be convinced efficiently while demonstrating the actor's decision-making abilities. The actor seems more believable if the audience can see it doing a fair amount of thinking (and particularly what it is thinking about) before performing an action.

Correct performance of the traditional story: Did the actor perform the story events in the order dictated by the traditional story? This is related to the performance criteria mentioned before. The actor simply is able to perform the single story in the desired order of events or not. 
Correct performance of the modified story without modification: Was the actor able to adapt to a different story within the same universe without requiring any modifications to its internal decision-making process? This is related to the design and reusability criteria mentioned before. If the actor can be used in different stories without needing to be extensively modified, it demonstrates a modularity of design between the virtual actor and its environment and thus can be easily reused.

Each virtual actor was placed within the traditional Goldilocks and the Three Bears story three times while recording how many times each decision had to be reevaluated and whether the actor performed the story correctly, then the same was done with the modified version of the story as well without any modification to the actor. Figure 5 shows the order that the actor has to interact with goal-satisfying locations in order to perform the two stories:

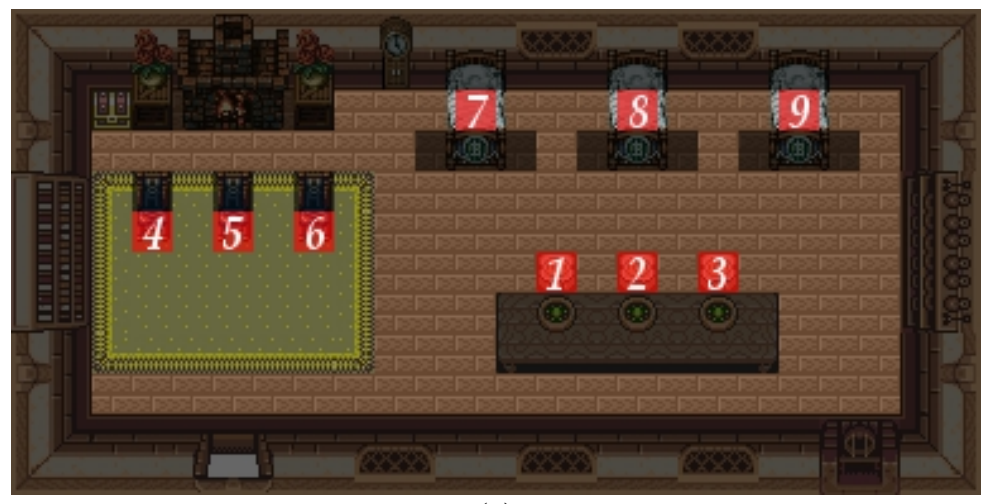

(a) 


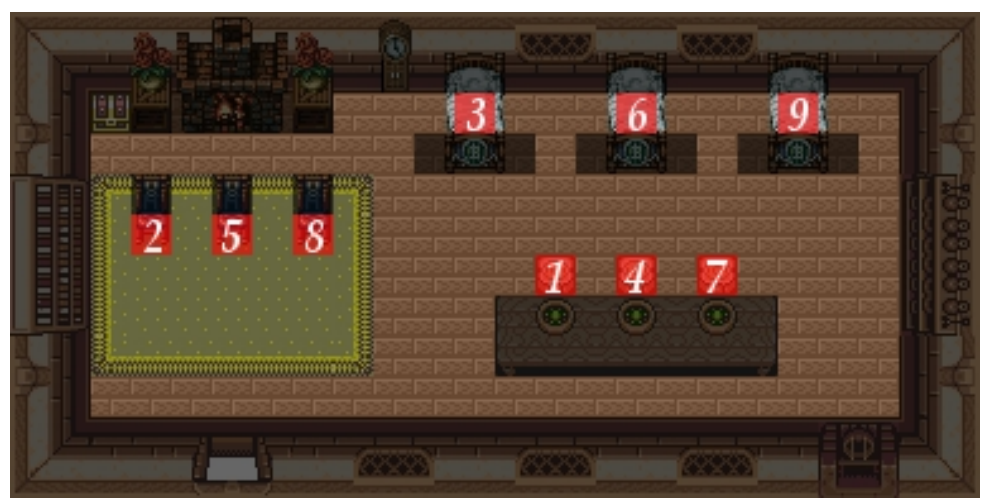

(b)

Figure 5: Story event order in the traditional and modified stories.

(a) The order that the virtual actor has to interact with goal-satisfying locations in order to perform the traditional Goldilocks and the Three Bears story.

(b) The order that the virtual actor has to interact with goal-satisfying locations in order to perform the modified Goldilocks and the Three Bears story.

As a note, the number of reevaluations done before making a decision is based on the particular implementation of the environment used in this demonstration. In particular, the autonomous actor's "desires" increase by a random amount between 1 and 10 every four seconds. This four second period will be referred to as a cycle. Also, each goal-satisfying node has an initial perceived satisfiability of 20 points and the story manager increases or decreases a goal-satisfying node by 5 points when a reevaluation is needed. Finally, the "desire" combined with the perceived satisfiability of the most satisfying location must be above 50 points before the autonomous actor chooses to satisfy the goal. When the goal is satisfied, the desire is always reduced by 20 points despite what the perceived satisfiability was.

Because of this, the results that follow can be viewed relative to the implementation. For example, the change in perceived satisfiability is $25 \%$ of the default value, thus the number of reevaluations would be around four times as many than an implementation that changed the perceived satisfiability by $100 \%$ of the default value. The change in perceived satisfiability in this implementation was chosen to be a small percentage of the default value so that the actor would better demonstrate the interplay between the actor and the story manager's manipulation of the environment by showing the internal debate between the actor's "desires" and the actor's perception of satisfiability. For a deployable application using environmental manipulation, the percentage of change should be adjusted to a suitable amount in order to not require excessive reevaluations. 


\section{Evaluating the Virtual Actors}

\section{The Scripted Virtual Actor: Traditional Story}

The results for the scripted virtual actor performing the traditional version of Goldilocks and the Three Bears are as follows:

\begin{tabular}{|c|c|c|}
\hline $\begin{array}{c}\text { Order in Which Story } \\
\text { Events Were Performed }\end{array}$ & Performed in Story Order & $\begin{array}{c}\text { Number of Reevaluations } \\
\text { Before Making Decision }\end{array}$ \\
\hline Eat Papa Bear's Porridge & Yes & 0 \\
\hline Eat Mama Bear's Porridge & Yes & 0 \\
\hline Eat Baby Bear's Porridge & Yes & 0 \\
\hline Sit in Papa Bear's Chair & Yes & 0 \\
\hline Sit in Mama Bear's Chair & Yes & 0 \\
\hline Sit in Baby Bear's Chair & Yes & 0 \\
\hline Sleep in Papa Bear's Bed & Yes & 0 \\
\hline Sleep in Mama Bear's Bed & Yes & 0 \\
\hline Sleep in Baby Bear's Bed & Yes & 0 \\
\hline
\end{tabular}

Table 2: Results of tests $1-\mathbf{3}$ for the scripted virtual actor performing the traditional story.

Since the scripted virtual actor by definition knows the locations and order of story events to perform, the results for the scripted actor were expected and were the same for all three tests. The actor went immediately from the starting location to Papa Bear's porridge, then Mama Bear's, and so on. No decision had to be reevaluated because the actor merely had to check the "script" for the next place to go; it has neither need nor ability to decide otherwise. Because of this, the actor was able to correctly perform the traditional version of the story with no difficulty.

The effect this has on believability is that the viewer does not see any type of decision-making process when the scripted actor moves from one location to the next. The movement from story event to story event is smooth and clean; there is no choice, so there is no decision to be made. Because the actor is not making any choices, the actor seems almost robotic in its behavior.

\section{The Scripted Virtual Actor: Modified Story}

The results for the scripted virtual actor performing the modified version of Goldilocks and the 
Three Bears are as follows:

\begin{tabular}{|c|c|c|}
\hline $\begin{array}{c}\text { Order in Which Story } \\
\text { Events Were Performed }\end{array}$ & Performed in Story Order & $\begin{array}{c}\text { Number of Reevaluations } \\
\text { Before Making Decision }\end{array}$ \\
\hline Eat Papa Bear's Porridge & Yes & 0 \\
\hline Sit in Papa Bear's Chair & Yes & 0 \\
\hline Sleep in Papa Bear's Bed & Yes & 0 \\
\hline Eat Mama Bear's Porridge & Yes & 0 \\
\hline Sit in Mama Bear's Chair & Yes & 0 \\
\hline Sleep in Mama Bear's Bed & Yes & 0 \\
\hline Eat Baby Bear's Porridge & Yes & 0 \\
\hline Sit in Baby Bear's Chair & Yes & 0 \\
\hline Sleep in Baby Bear's Bed & Yes & 0 \\
\hline
\end{tabular}

Table 3: Results of tests $1-3$ for the scripted virtual actor performing the modified story.

Since the modified story existed in the same environment and it was explicitly given the modified story event order, the scripted actor was able to perform the modified script perfectly, again only making a single decision each time. This and the previous test establish a base line for comparison with the autonomous virtual actor with and without environmental manipulation.

\section{The Autonomous Virtual Actor without Environmental Manipulation: Traditional Story}

The results for the autonomous virtual actor without environmental manipulation performing the traditional version of Goldilocks and the Three Bears are as follows: 


\begin{tabular}{|c|c|c|}
\hline $\begin{array}{c}\text { Order in Which Story } \\
\text { Events Were Performed }\end{array}$ & Performed in Story Order & $\begin{array}{c}\text { Number of Reevaluations } \\
\text { Before Making Decision }\end{array}$ \\
\hline Sleep in Baby Bear's Bed & No & 0 \\
\hline Sit in Papa Bear's Chair & No & 0 \\
\hline Sleep in Mama Bear's Bed & No & 0 \\
\hline Eat Papa Bear's Porridge & No & 0 \\
\hline Sit in Baby Bear's Chair & No & 0 \\
\hline Sleep in Papa Bear's Bed & No & 0 \\
\hline Eat Baby Bear's Porridge & No & 0 \\
\hline Sit in Mama Bear's Chair & No & 0 \\
\hline Eat Mama Bear's Porridge & No & 0 \\
\hline
\end{tabular}

Table 4: Results of test 1 for the autonomous virtual actor without environmental manipulation performing the traditional story.

\begin{tabular}{|c|c|c|}
\hline $\begin{array}{c}\text { Order in Which Story } \\
\text { Events Were Performed }\end{array}$ & Performed in Story Order & $\begin{array}{c}\text { Number of Reevaluations } \\
\text { Before Making Decision }\end{array}$ \\
\hline Sit in Baby Bear's Chair & No & 0 \\
\hline Sleep in Mama Bear's Bed & No & 0 \\
\hline Eat Baby Bear's Porridge & Yes & 0 \\
\hline Sit in Mama Bear's Chair & No & 0 \\
\hline Sleep in Baby Bear's Bed & No & 0 \\
\hline Sleep in Papa Bear's Bed & No & 0 \\
\hline Sit in Papa Bear's Chair & No & 0 \\
\hline Eat Mama Bear's Porridge & No & 0 \\
\hline Eat Papa Bear's Porridge & No & 0 \\
\hline
\end{tabular}

Table 5: Results of test 2 for the autonomous virtual actor without environmental manipulation performing the traditional story.

\begin{tabular}{|c|c|c|}
\hline $\begin{array}{c}\text { Order in Which Story } \\
\text { Events Were Performed }\end{array}$ & Performed in Story Order & $\begin{array}{c}\text { Number of Reevaluations } \\
\text { Before Making Decision }\end{array}$ \\
\hline Sleep in Papa Bear's Bed & No & 0 \\
\hline Eat Papa Bear's Porridge & No & 0 \\
\hline Eat Mama Bear's Porridge & No & 0 \\
\hline Sit in Papa Bear's Chair & Yes & 0 \\
\hline Sleep in Mama Bear's Bed & No & 0 \\
\hline Eat Baby Bear's Porridge & No & 0 \\
\hline Sit in Baby Bear's Chair & No & 0 \\
\hline
\end{tabular}




\begin{tabular}{|c|c|c|}
\hline $\begin{array}{c}\text { Order in Which Story } \\
\text { Events Were Performed }\end{array}$ & Performed in Story Order & $\begin{array}{c}\text { Number of Reevaluations } \\
\text { Before Making Decision }\end{array}$ \\
\hline Sleep in Baby Bear's Bed & No & 0 \\
\hline Sit in Mama Bear's Chair & No & 0 \\
\hline
\end{tabular}

Table 6: Results of test 3 for the autonomous virtual actor without environmental manipulation performing the traditional story.

Since the autonomous virtual actor behaves by choosing the best means to satisfy its goals and the amount of "desire" to satisfy a goal is based on a random factor, the overall behavior of the actor cannot be assumed. This can be seen in the results of the three tests with the autonomous actor in which while some story events were performed at the correct time, none of the three stories performed by the actor match the story that was hoped to be obtained. In fact, there is only a $1 /(9 !)$ chance $(0.000276 \%)$ of the autonomous actor ever performing the traditional story because of its random nature.

In addition, since the autonomous actor has no means of feedback and the environment does not respond to its decision in this case, there is no need for the actor to reevaluate its decisions. However, we do notice some delay before a few decisions are made. The first decision made by the autonomous actor takes as long as it does because the actor does not need to make a decision immediately; no goals need satisfying at the moment. A decision will only be made once the "desire" to satisfy a goal exists and a goal-satisfying location's perceived satisfiability are high enough to warrant any change in behavior. Also, delays after the first are because after some satisfaction, the actor's goals may again be below the threshold that provokes the actor to make a decision.

Because the autonomous actor's decision-making process can be seen through both the delays before the actor makes a decision and the ability of the actor to display its decision-making process, the autonomous actor seems much more believable than the scripted actor. While the actor will not perform the desired story reliably, the fact that it is a decision-making organism grants it more potential for believability because we can see it think and intelligently handle making choices.

\section{The Autonomous Virtual Actor without Environmental Manipulation: Modified Story}

The results for the autonomous virtual actor without environmental manipulation performing the modified version of Goldilocks and the Three Bears are as follows: 


\begin{tabular}{|c|c|c|}
\hline $\begin{array}{c}\text { Order in Which Story } \\
\text { Events Were Performed }\end{array}$ & Performed in Story Order & $\begin{array}{c}\text { Number of Reevaluations } \\
\text { Before Making Decision }\end{array}$ \\
\hline Sleep in Baby Bear's Bed & No & 0 \\
\hline Sleep in Mama Bear's Bed & No & 0 \\
\hline Sit in Baby Bear's Chair & No & 0 \\
\hline Eat Mama Bear's Porridge & Yes & 0 \\
\hline Sleep in Papa Bear's Bed & No & 0 \\
\hline Eat Papa Bear's Porridge & No & 0 \\
\hline Sit in Papa Bear's Chair & No & 0 \\
\hline Eat Baby Bear's Porridge & No & 0 \\
\hline Sit in Mama Bear's Chair & No & 0 \\
\hline
\end{tabular}

Table 7: Results of test 1 for the autonomous virtual actor without environmental manipulation performing the modified story.

\begin{tabular}{|c|c|c|}
\hline $\begin{array}{c}\text { Order in Which Story } \\
\text { Events Were Performed }\end{array}$ & Performed in Story Order & $\begin{array}{c}\text { Number of Reevaluations } \\
\text { Before Making Decision }\end{array}$ \\
\hline Eat Baby Bear's Porridge & No & 0 \\
\hline Sleep in Papa Bear's Bed & No & 0 \\
\hline Sit in Papa Bear's Chair & No & 0 \\
\hline Eat Mama Bear's Porridge & Yes & 0 \\
\hline Sleep in Mama Bear's Bed & No & 0 \\
\hline Sit in Mama Bear's Chair & No & 0 \\
\hline Eat Papa Bear's Porridge & No & 0 \\
\hline Sit in Baby Bear's Chair & Yes & 0 \\
\hline Sleep in Baby Bear's Bed & Yes & 0 \\
\hline
\end{tabular}

Table 8: Results of test 2 for the autonomous virtual actor without environmental manipulation performing the modified story.

\begin{tabular}{|c|c|c|}
\hline $\begin{array}{c}\text { Order in Which Story } \\
\text { Events Were Performed }\end{array}$ & Performed in Story Order & $\begin{array}{c}\text { Number of Reevaluations } \\
\text { Before Making Decision }\end{array}$ \\
\hline Sleep in Baby Bear's Bed & No & 0 \\
\hline Sit in Baby Bear's Chair & No & 0 \\
\hline Eat Baby Bear's Porridge & No & 0 \\
\hline Sleep in Papa Bear's Bed & No & 0 \\
\hline Sit in Mama Bear's Chair & Yes & 0 \\
\hline Eat Papa Bear's Porridge & No & 0 \\
\hline Sleep in Mama Bear's Bed & No & 0 \\
\hline
\end{tabular}




\begin{tabular}{|c|c|c|}
\hline $\begin{array}{c}\text { Order in Which Story } \\
\text { Events Were Performed }\end{array}$ & Performed in Story Order & $\begin{array}{c}\text { Number of Reevaluations } \\
\text { Before Making Decision }\end{array}$ \\
\hline Eat Mama Bear's Porridge & No & 0 \\
\hline Sit in Papa Bear's Chair & No & 0 \\
\hline
\end{tabular}

Table 9: Results of test 3 for the autonomous virtual actor without environmental manipulation performing the modified story.

Again, we see similar results as in the first test, which is to be expected since the autonomous actor has no bias towards any one particular story because of its random nature; it simply does what it wants to. Because of this, the issue of reusability has two sides to it: how reusable the autonomous actor is in terms of intelligently behaving in a different environment and how reusable the actor is in terms of telling the correct story.

In the first case, the autonomous actor has the capability to intelligently analyze the environment and perform decision-making even when placed in a different environment. When the story begins, the autonomous actor seeks out the goal-satisfying locations in the environment, so if these locations were moved somewhere else in the environment, the actor would still be able to find them and move to their locations to satisfy its goals when needed.

In terms of performing different stories, however, the reusability of the actor is not even applicable since, as we have seen in the actor's performance of the traditional and modified stories, it is highly unlikely that the autonomous actor would correctly perform any of the stories correctly. As mentioned in the previous example, the $0.000276 \%$ chance of correctly performing the desired story means that the autonomous actor cannot be expected to even be able to tell a single story in the first place, let alone be reliably reusable for multiple stories.

\section{The Autonomous Virtual Actor with Environmental Manipulation: Traditional Story}

The results for the autonomous virtual actor with environmental manipulation performing the modified version of Goldilocks and the Three Bears are as follows:

\begin{tabular}{|c|c|c|}
\hline $\begin{array}{c}\text { Order in Which Story } \\
\text { Events Were Performed }\end{array}$ & $\begin{array}{c}\text { Performed in Correct } \\
\text { Story Order }\end{array}$ & $\begin{array}{c}\text { Number of Reevaluations } \\
\text { Before Making Decision }\end{array}$ \\
\hline Eat Papa Bear's Porridge & Yes & 3 \\
\hline
\end{tabular}




\begin{tabular}{|c|c|c|}
\hline $\begin{array}{c}\text { Order in Which Story } \\
\text { Events Were Performed }\end{array}$ & $\begin{array}{c}\text { Performed in Correct } \\
\text { Story Order }\end{array}$ & $\begin{array}{c}\text { Number of Reevaluations } \\
\text { Before Making Decision }\end{array}$ \\
\hline Eat Mama Bear's Porridge & Yes & 8 \\
\hline Eat Baby Bear's Porridge & Yes & 13 \\
\hline Sit in Papa Bear's Chair & Yes & 5 \\
\hline Sit in Mama Bear's Chair & Yes & 13 \\
\hline Sit in Baby Bear's Chair & Yes & 1 \\
\hline Sleep in Papa Bear's Bed & Yes & 1 \\
\hline Sleep in Mama Bear's Bed & Yes & 0 \\
\hline Sleep in Baby Bear's Bed & Yes & \\
\hline
\end{tabular}

Table 10: Results of test 1 for the autonomous virtual actor with environmental manipulation performing the traditional story.

\begin{tabular}{|c|c|c|}
\hline $\begin{array}{c}\text { Order in Which Story } \\
\text { Events Were Performed }\end{array}$ & $\begin{array}{c}\text { Performed in Correct } \\
\text { Story Order }\end{array}$ & $\begin{array}{c}\text { Number of Reevaluations } \\
\text { Before Making Decision }\end{array}$ \\
\hline Eat Papa Bear's Porridge & Yes & 2 \\
\hline Eat Mama Bear's Porridge & Yes & 6 \\
\hline Eat Baby Bear's Porridge & Yes & 0 \\
\hline Sit in Papa Bear's Chair & Yes & 1 \\
\hline Sit in Mama Bear's Chair & Yes & 4 \\
\hline Sit in Baby Bear's Chair & Yes & 1 \\
\hline Sleep in Papa Bear's Bed & Yes & 0 \\
\hline Sleep in Mama Bear's Bed & Yes & 0 \\
\hline Sleep in Baby Bear's Bed & Yes & 12 \\
\hline
\end{tabular}

Table 11: Results of test 2 for the autonomous virtual actor with environmental manipulation performing the traditional story.

\begin{tabular}{|c|c|c|}
\hline $\begin{array}{c}\text { Order in Which Story } \\
\text { Events Were Performed }\end{array}$ & $\begin{array}{c}\text { Performed in Correct } \\
\text { Story Order }\end{array}$ & $\begin{array}{c}\text { Number of Reevaluations } \\
\text { Before Making Decision }\end{array}$ \\
\hline Eat Papa Bear's Porridge & Yes & 0 \\
\hline Eat Mama Bear's Porridge & Yes & 2 \\
\hline Eat Baby Bear's Porridge & Yes & 7 \\
\hline Sit in Papa Bear's Chair & Yes & 5 \\
\hline Sit in Mama Bear's Chair & Yes & 9 \\
\hline Sit in Baby Bear's Chair & Yes & 0 \\
\hline Sleep in Papa Bear's Bed & Yes & 1 \\
\hline Sleep in Mama Bear's Bed & Yes & 0 \\
\hline
\end{tabular}




\begin{tabular}{|c|c|c|}
\hline $\begin{array}{c}\text { Order in Which Story } \\
\text { Events Were Performed }\end{array}$ & $\begin{array}{c}\text { Performed in Correct } \\
\text { Story Order }\end{array}$ & $\begin{array}{c}\text { Number of Reevaluations } \\
\text { Before Making Decision }\end{array}$ \\
\hline Sleep in Baby Bear's Bed & Yes & 0 \\
\hline
\end{tabular}

Table 12: Results of test 3 for the autonomous virtual actor with environmental manipulation performing the traditional story.

The first thing to notice from these results is that despite the virtual actor being autonomous and thus primarily controlled by some factor of randomness, the actor performed the traditional story for Goldilocks and the Three Bears correctly all three times. The story manager was able to slowly convince the autonomous actor to perform the required story events in order to tell the traditional story. Figure 6 shows an example of this in which the autonomous actor is choosing to eat Baby Bear's porridge despite the fact that its hunger did not need to be satisfied as much as its need for comfort and sleep:

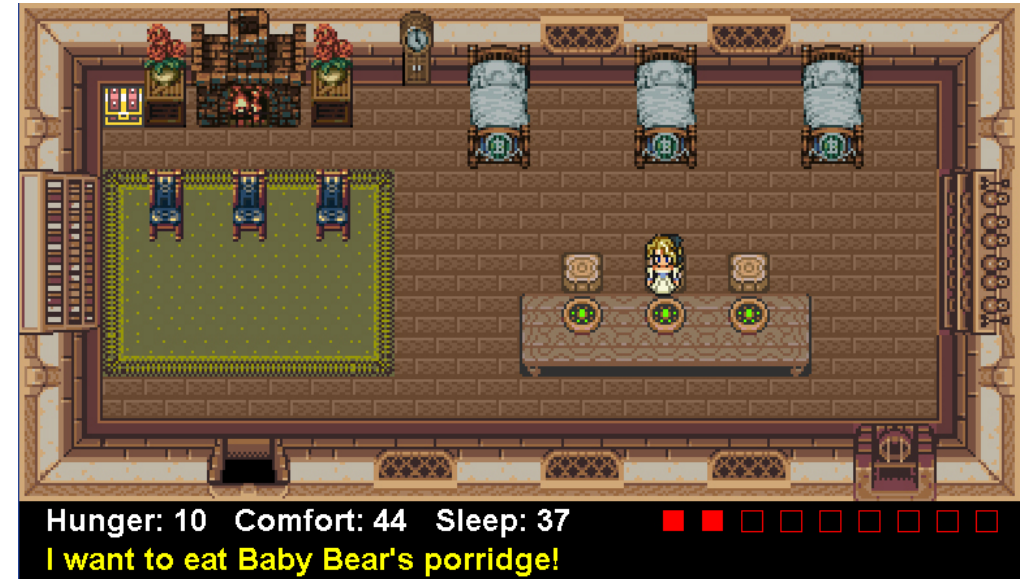

Figure 6: Screenshot of virtual actor being influenced towards a story event.

The story manager manipulates the environment in order to convince the autonomous virtual actor to perform the next story event in the story. In this figure, the actor has chosen to eat even though its need for comfort and sleep is significantly higher than its hunger.

We can also see the effect of the story manager's interplay in the number of reevaluations of the actor's decisions before it chose the desired behavior. The actor had to be increasingly provoked in order to perform three story events in a row that satisfied the same goal each time, which caused the number of reevaluations to increase for choosing to eat porridge and sit in the chairs in the right order. An example of this interplay is the following: 
Next Story Event: Eat Baby Bear's Porridge

Current Values: Hunger (10), Comfort (44), Sleep (37), All Goal-Satisfying Locations (20)

Autonomous Actor (AA): I want to sit on Baby Bear's chair!

Story Manager (SM): Increasing perceived satisfaction of Baby Bear's porridge to 20

SM: Decreasing perceived satisfaction of Baby Bear's chair to 10

AA: But Baby Bear's porridge looks very tasty...

AA: I want to sit on Mama Bear's chair!

SM: Increasing perceived satisfaction of Baby Bear's porridge to 25

SM: Decreasing perceived satisfaction of Mama Bear's chair to 10

AA: But Baby Bear's porridge looks very tasty...

AA: I want to sit on Papa Bear's chair!

SM: Increasing perceived satisfaction of Baby Bear's porridge to 30

SM: Decreasing perceived satisfaction of Papa Bear's chair to 10

AA: But Baby Bear's porridge looks very tasty...

AA: I want to sleep in Baby Bear's bed!

SM: Increasing perceived satisfaction of Baby Bear's porridge to 35

SM: Decreasing perceived satisfaction of Baby Bear's bed to 15

AA: But Baby Bear's porridge looks very tasty...

AA: I want to sleep in Mama Bear's bed!

SM: Increasing perceived satisfaction of Baby Bear's porridge to 40

SM: Decreasing perceived satisfaction of Mama Bear's bed to 15

AA: But Baby Bear's porridge looks very tasty...

AA: I want to sleep in Papa Bear's bed!

SM: Increasing perceived satisfaction of Baby Bear's porridge to 45

SM: Decreasing perceived satisfaction of Papa Bear's bed to 15

AA: But Baby Bear's porridge looks very tasty...

AA: I want to eat Baby Bear's porridge!

In addition, because of the interplay between the story manager and the actor's decision-making

process, the actor took a little bit longer to make some of its decisions, especially after having satisfied a

goal several times and being influenced to satisfy it again. Even in this simple example, this delay in

making a decision gives the actor more potential believability than the scripted actor because this actor is

spending some time analyzing multiple factors before making a final decision that can be exposed to the

audience through Goldilocks' body language and speech. This process is reminiscent of Tevye in Fiddler

on the Roof, when deciding what to do about an untraditional match for his daughter:

“...But what kind of match would that be, with a poor tailor?

On the other hand, he's an honest, hard worker.

On the other hand, he has absolutely nothing.

On the other hand, things could never get worse for him, they could only get better...” [Fiddler]

The autonomous actor is going through a similar internal debate while the story manager is influencing the actor through the environment. If more detailed internal dialogue was implemented and was viewable, an 
example of this internal debate could have been the following:

"I would like to take a nap in Papa Bear's bed...

but Baby Bear's porridge looks kind of appetizing...

but I am really tired...

but that bed doesn't look all that comfortable, and that porridge looks awfully good...

but it's getting hard to keep my eyes open...

but I guess I have enough energy to eat that yummy porridge."

As a note, because the environment only lets a goal-satisfying location be used once in the story, the use of the Bears' beds did not require more than one reevaluation of a decision because the only goal that could be satisfied at that point in the story was the need for sleep. Any reevaluation was the result of the story manager simply influencing the actor to the right bed to sleep on.

\section{The Autonomous Virtual Actor with Environmental Manipulation: Modified Story}

The results for the autonomous virtual actor with environmental manipulation performing the modified version of Goldilocks and the Three Bears are as follows:

\begin{tabular}{|c|c|c|}
\hline $\begin{array}{c}\text { Order in Which Story } \\
\text { Events Were Performed }\end{array}$ & $\begin{array}{c}\text { Performed in Correct } \\
\text { Story Order }\end{array}$ & $\begin{array}{c}\text { Number of Reevaluations } \\
\text { Before Making Decision }\end{array}$ \\
\hline Eat Papa Bear's Porridge & Yes & 2 \\
\hline Sit in Papa Bear's Chair & Yes & 1 \\
\hline Sleep in Papa Bear's Bed & Yes & 1 \\
\hline Eat Mama Bear's Porridge & Yes & 3 \\
\hline Sit in Mama Bear's Chair & Yes & 0 \\
\hline Sleep in Mama Bear's Bed & Yes & 4 \\
\hline Eat Baby Bear's Porridge & Yes & 2 \\
\hline Sit in Baby Bear's Chair & Yes & 0 \\
\hline Sleep in Baby Bear's Bed & Yes & 4 \\
\hline
\end{tabular}

Table 13: Results of test 1 for the autonomous virtual actor with environmental manipulation performing the modified story.

\begin{tabular}{|c|c|c|}
\hline $\begin{array}{c}\text { Order in Which Story } \\
\text { Events Were Performed }\end{array}$ & $\begin{array}{c}\text { Performed in Correct } \\
\text { Story Order }\end{array}$ & $\begin{array}{c}\text { Number of Reevaluations } \\
\text { Before Making Decision }\end{array}$ \\
\hline Eat Papa Bear's Porridge & Yes & 1 \\
\hline Sit in Papa Bear's Chair & Yes & 1 \\
\hline Sleep in Papa Bear's Bed & Yes & 1 \\
\hline Eat Mama Bear's Porridge & Yes & 1 \\
\hline
\end{tabular}




\begin{tabular}{|c|c|c|}
\hline $\begin{array}{c}\text { Order in Which Story } \\
\text { Events Were Performed }\end{array}$ & $\begin{array}{c}\text { Performed in Correct } \\
\text { Story Order }\end{array}$ & $\begin{array}{c}\text { Number of Reevaluations } \\
\text { Before Making Decision }\end{array}$ \\
\hline Sit in Mama Bear's Chair & Yes & 1 \\
\hline Sleep in Mama Bear's Bed & Yes & 1 \\
\hline Eat Baby Bear's Porridge & Yes & 1 \\
\hline Sit in Baby Bear's Chair & Yes & 0 \\
\hline Sleep in Baby Bear's Bed & Yes & 0 \\
\hline
\end{tabular}

Table 14: Results of test 2 for the autonomous virtual actor with environmental manipulation performing the modified story.

\begin{tabular}{|c|c|c|}
\hline $\begin{array}{c}\text { Order in Which Story } \\
\text { Events Were Performed }\end{array}$ & $\begin{array}{c}\text { Performed in Correct } \\
\text { Story Order }\end{array}$ & $\begin{array}{c}\text { Number of Reevaluations } \\
\text { Before Making Decision }\end{array}$ \\
\hline Eat Papa Bear's Porridge & Yes & 1 \\
\hline Sit in Papa Bear's Chair & Yes & 1 \\
\hline Sleep in Papa Bear's Bed & Yes & 1 \\
\hline Eat Mama Bear's Porridge & Yes & 2 \\
\hline Sit in Mama Bear's Chair & Yes & 1 \\
\hline Sleep in Mama Bear's Bed & Yes & 1 \\
\hline Eat Baby Bear's Porridge & Yes & 0 \\
\hline Sit in Baby Bear's Chair & Yes & 0 \\
\hline Sleep in Baby Bear's Bed & Yes & \\
\hline
\end{tabular}

Table 15: Results of test 3 for the autonomous virtual actor with environmental manipulation performing the modified story.

Again, it should be noted that the autonomous actor performed the modified Goldilocks and the

Three Bears story correctly in all three cases. Not only are the performance and believability criteria met, as described in the previous case, this test shows how the autonomous actor can be reused without modification for different stories and correctly perform its role with the influence of the story manager; all that changed between these two stories was the order of the story events given to the story manager.

As a note, the number of reevaluations in the modified story was much lower because this story catered to fulfilling all of the goals evenly instead of all of one type at a time. The traditional story requires that the actor satisfy the same goal three times in a row, but the modified story allows the actor to eat, then sit, then sleep before repeating the order, which allows for the goals to naturally increase and become candidates for the actor to want to satisfy without much influence. 
Since the modified story has no unique quality about the ordering of the events - just that they are ordered differently than the traditional story - it stands to reason than any particular ordering of the Goldilocks and the Three Bears story events will demonstrate the adaptability of the autonomous actor. Further modifications such as performing the traditional story in backwards order produce similar results in terms of having several reevaluations to guide the autonomous actor towards correctly performing the desired story.

\section{Using Environmental Manipulation for Multiple Event-Based Stories}

By demonstrating the capabilities of environmental manipulation to influence an autonomous actor to perform a single story, the foundation for performing multiple event-based stories has been laid. Because event-based storytelling with multiple stories allows virtual actors to proceed down different paths in a story event graph, the story manager can be enhanced to guide the actor to the next appropriate story event. When the story manager knows that the virtual actor should make a decision between one of many different story paths, the story manager could use a heuristic to determine the actor's "proximity" to a particular path. For example, if a virtual actor needed to make the decision to travel to a destination through either a mountain trail, a forest path, or an open plain, the story manager could take into account multiple factors such as the physical proximity of the actor to each path, the capabilities of the actor in terms of traveling in each type of landscape, or the actor's affinity towards a particular journey depending on its past experiences. Together, these factors can serve as a heuristic that lets the story manager know in what direction to influence the actor. Additionally, the story manager could simply allow the autonomy of the virtual actor to determine the chosen path.

To demonstrate multiple event-based stories, the story manager was modified to use the environment to influence Goldilocks to eat any porridge, and then whichever porridge Goldilocks chooses by her own decision, the story manager will then influence her to sit in the chair and sleep in the bed, in that order, that is owned by the same bear as the porridge she ate. For example, if Goldilocks chooses to eat Mama Bear's porridge, the story manager then will influence her to sit in Mama Bear's chair and then sleep in Mama Bear's bed, at which time the story ends. The story manager does this by having three event-based stories with three story events each, one with Papa Bear's porridge, chair, and bed, another 
with Mama Bear's porridge, chair, and bed, and another with Baby Bear's porridge, chair, and bed as the multiple storylines. When Goldilocks decides on an action, the story manager compares her decision with all three stories' porridge events, and if that decision does not match with any of the three stories' porridge events, the story manager increases the perceived satisfaction of all three porridges and decreases her chosen goal satisfying location (as was previously being done). By increasing the perceived satisfaction of all three porridges equally, it makes Goldilocks' chosen storyline unbiased. Once Goldilocks eats a particular bowl of porridge, the story manager chooses the corresponding storyline as the "main" storyline and then proceeds to do what it does in the single event-based storyline demonstration for the remaining story events. The demonstration randomly starts one of the three storylines when Goldilocks finally decides on which porridge to eat, but reliably finishes the storyline that corresponds with her decision.

Similar to the single event-based story tests performed above, three tests were run with the multiple event-based stories. The results for the autonomous virtual actor with environmental manipulation performing the multiple event-based stories version of Goldilocks and the Three Bears are as follows:

\begin{tabular}{|c|c|c|}
\hline $\begin{array}{c}\text { Order in Which Story } \\
\text { Events Were Performed }\end{array}$ & $\begin{array}{c}\text { Performed in Correct } \\
\text { Story Order }\end{array}$ & $\begin{array}{c}\text { Number of Reevaluations } \\
\text { Before Making Decision }\end{array}$ \\
\hline Eat Baby Bear's Porridge & N/A & 3 \\
\hline Sit in Baby Bear's Chair & Yes & 1 \\
\hline Sleep in Baby Bear's Bed & Yes & 1 \\
\hline
\end{tabular}

Table 16: Results of test 1 for the autonomous virtual actor with environmental manipulation performing one of the multiple event-based stories.

\begin{tabular}{|c|c|c|}
\hline $\begin{array}{c}\text { Order in Which Story } \\
\text { Events Were Performed }\end{array}$ & $\begin{array}{c}\text { Performed in Correct } \\
\text { Story Order }\end{array}$ & $\begin{array}{c}\text { Number of Reevaluations } \\
\text { Before Making Decision }\end{array}$ \\
\hline Eat Mama Bear's Porridge & N/A & 0 \\
\hline Sit in Mama Bear's Chair & Yes & 1 \\
\hline Sleep in Mama Bear's Bed & Yes & 0 \\
\hline
\end{tabular}

Table 17: Results of test 2 for the autonomous virtual actor with environmental manipulation performing one of the multiple event-based stories.

\begin{tabular}{|c|c|c|}
\hline $\begin{array}{c}\text { Order in Which Story } \\
\text { Events Were Performed }\end{array}$ & $\begin{array}{c}\text { Performed in Correct } \\
\text { Story Order }\end{array}$ & $\begin{array}{c}\text { Number of Reevaluations } \\
\text { Before Making Decision }\end{array}$ \\
\hline Eat Papa Bear's Porridge & N/A & 3 \\
\hline
\end{tabular}




\begin{tabular}{|c|c|c|}
\hline $\begin{array}{c}\text { Order in Which Story } \\
\text { Events Were Performed }\end{array}$ & $\begin{array}{c}\text { Performed in Correct } \\
\text { Story Order }\end{array}$ & $\begin{array}{c}\text { Number of Reevaluations } \\
\text { Before Making Decision }\end{array}$ \\
\hline Sit in Papa Bear's Chair & Yes & 3 \\
\hline Sleep in Papa Bear's Bed & Yes & 1 \\
\hline
\end{tabular}

Table 18: Results of test 3 for the autonomous virtual actor with environmental manipulation performing one of the multiple event-based stories.

In all three tests, Goldilocks was influenced towards eating porridge as her first action, but she was able to randomly choose which porridge she wants to eat. Once a bowl of porridge was chosen, the story manager manipulated the environment to reliably convince Goldilocks' to sit in the chair and then sleep in the bed owned by the same bear as the one whose porridge she ate.

\section{Analyzing the Results}

From the results of this exploration, the use of scripting and autonomy without environmental manipulation were not able to meet all of the criteria judging reusability, correct story performance, and believability. However, the use of a manipulatable environment for advancing event-based stories proved capable in all of these areas: the virtual actor was able to perform the single and multiple event-based stories efficiently and correctly, was able to be reused in a different story because of its modular design, and had some elements of believability in its actions.

\section{COMPARISONS WITH OTHER STORYTELLING APPROACHES}

Table 19 compares storytelling with environmental manipulation with the other storytelling approaches discussed in this paper:

\begin{tabular}{|c|c|c|c|c|c|}
\hline & Scripting & Autonomy & $\begin{array}{c}\text { Black \& } \\
\text { White }\end{array}$ & Façade & $\begin{array}{c}\text { Environ. } \\
\text { Manipulation }\end{array}$ \\
\hline Design / Reusability & POOR & STRONG & UNKNOWN & UNKNOWN & STRONG \\
\hline Event-Based Story Performance & STRONG & POOR & MEDIUM & STRONG & STRONG \\
\hline Emergent Story Performance & POOR & STRONG & STRONG & MEDIUM & $\begin{array}{c}\text { FUTURE } \\
\text { WORK }\end{array}$ \\
\hline Believability & MEDIUM & STRONG & MEDIUM & MEDIUM & STRONG \\
\hline
\end{tabular}




\begin{tabular}{|c|c|c|c|c|c|}
\hline & Scripting & Autonomy & $\begin{array}{c}\text { Black \& } \\
\text { White }\end{array}$ & Façade & $\begin{array}{c}\text { Environ. } \\
\text { Manipulation }\end{array}$ \\
\hline Audience Interaction & POOR & STRONG & STRONG & MEDIUM & $\begin{array}{c}\text { FUTURE } \\
\text { WORK }\end{array}$ \\
\hline
\end{tabular}

Table 19: The strengths and weaknesses of environmental manipulation in terms of digital storytelling criteria compared with the other storytelling approaches.

As seen above, storytelling with environmental manipulation is able to perform strongly in the categories or reusability, correct event-based story performance, and believability, with potential for strength in emergent story performance and audience interaction. Because of the modularity of the virtual actor, the environment, and the story manager, the three entities are designed to support reusability, allowing the actor, the environment, and the story manager to be reused for different stories. This will be discussed further in the "Contributions" section. Also, virtual actors are able to perform both single and multiple event-based stories as discussed in the demonstration. In addition, believability is maintained because the actor is never controlled, but is allowed to come to the decision to follow a story, and because an autonomous virtual actor is used, the audience can be able to see an intelligent, decision-making character rather than a robotic entity that only does what it is told. The virtual actor can use body language and verbal messages to show the decision-making process it is going through, giving the actor many more dimensions than a scripted actor.

The divorce of the virtual actor from a story manager in this approach also promotes better design in comparison to the other storytelling approaches. In The Sciences of the Artificial, Herbert Simon writes:

An artifact can be thought of as a meeting point - an 'interface' in today's terms - between an 'inner' environment, the substance and organization of the artifact itself, and an 'outer' environment, the surroundings in which it operates...

Thus the first advantage of dividing outer from inner environment in studying an adaptive or artificial system is that we can often predict behavior from knowledge of the system's goals and its outer environment, with only minimal assumptions about the inner environment. An instant corollary is that we often find quite different inner environments accomplishing identical or similar goals in identical or similar outer environments - airplanes and birds, dolphins and tunafish, weight-driven clocks and spring-driven clocks, electrical relays and transistors. [Simon, pp. 9, 11]

Natural and man-made artifacts are entities in which the internal mechanisms of the artifact are usually only observable and interactive through an interface between the inner and outer environments. For 
example, animal behavior is primarily understood through observation of their actions in their environments, not through an autopsy of the animal's body. In this case, the body is too complex to reliably link behavior to biology, and internal inspection could lead to permanent changes in the animal's behavior, such as infection, disability, or death. Therefore, artificial intelligence design should likewise promote the separation of an actor's artificial intelligence and the environment in which it exists. The interaction between the actor's artificial intelligence and the environment should be only through an appropriate interface consisting of aspects of the actor's goals and behaviors. One particular demonstration of this interaction in this approach which does not exist in other approaches is between the story manager and the virtual actor. The story manager simply observes the decisions made by the virtual actor and acts accordingly to what the manager discovers. It does not, however, have access to the decision-making process of the actor or have the ability to explicitly control the behavior of the actor; to do so would violate the encapsulation of the design.

\section{CONTRIBUTIONS}

The use of completely autonomous virtual actors, a manipulatable environment, and an independent story manager offers an important contribution to the field of digital storytelling: a novel artificial intelligence architecture for storytelling that enables reusable artificial intelligence components and achieves better believability of the virtual actors while achieving specific story goals. Because of the modular nature of all three components, these components can be easily reusable, saving the trouble of creating entirely new artificial intelligence architectures for every new storytelling application. A similar example is with the Havok physics engine. The creators of Havok were able to focus on the development and improvement of physics for video games independent of how it would be used in the games. Such games as Half-Life 2 have been greatly enhanced by such an impressive physics engine without having to "reinvent the wheel" of physics for each game [Havok]. This can be the case for artificial intelligence design as well. The development of complex, versatile, and believable autonomous actors as well as an architecture for an adaptable environment and story manager can take place independent of the game engine and what type of storytelling the components will be used for. For example, such a virtual actor could be placed on a farm and given a hoe, and with the help of the environment the actor would be able to 
be used in a story in which the actor is a farmer. In addition, the same virtual actor could be placed on a battle field and given a rifle, and the actor would be able to be used in a story as a soldier. In order to easily adapt to each different scenario, the virtual actors could have some personality parameters that can be initially customized, but afterwards the internal mechanisms of the actor and the interaction with the environment would be able to perform any story without a story manager explicitly giving commands to the actor. This can be realized either within a particular game development company in that they can invest in reusable artificial intelligence development in-house, saving money and man hours over the course of several game development cycles, or realized as a library of artificial intelligence tools for any developer to use, which will be especially useful for the growing number of independent game developers entering the gaming industry.

This storytelling architecture also addresses some of the problems with current methods in regards to the believability of the virtual actors. Because the actors are autonomous, these actors will respond appropriately to unforeseen situations through reactive and adaptive behavior, which scripted actors are unable to do, thus hindering the believability of the scripted actors' actions. These autonomous actors can be implemented to be able to express their reactions and thoughts in ways that scripted actors cannot because they behave according to what they perceive and what they desire rather than specific actions. With the help of the story manager, these autonomous actors can be influenced to align their desires with the story being performed, and because the actor chooses to follow the story rather than being coerced, its believability is not hindered by any direct control from external entities.

Additionally, using autonomous virtual actors and environmental manipulation allows for game developers to be able to rely on a consistent storytelling performance. Reliable storytelling is an important aspect of video game storytelling since most games have a particular story to be told. Video game developers want to ensure that the story is performed appropriately on every player's machine, since at that point it is obviously out of their control, and have had to sacrifice some believability of their actors by relying on scripted artificial intelligence as a story-driving mechanism. However, the story manager's ability to influence the actor towards the necessary story events by manipulating the environment around the actor ensures that the correct performance of the story will be reliably achieved while preserving the believability of the actor by not directly controlling its behavior. 
Finally, the environmental manipulation storytelling approach contributes new research into the relatively scarce amount of research within the realm of digital storytelling. As mentioned before, Michael Mateas stated that little work has been done to combine autonomous, believable virtual actors with a story's more deliberate plot. This new method of digital storytelling provides another step into that realm and provides another path for future research.

\section{FUTURE WORK}

While it was demonstrated that digital storytelling with environmental manipulation could reliably perform event-based stories while maintaining believability and promoting reusability, this storytelling approach can be further enhanced for the performance of emergent stories and to allow for audience interaction. The story manager's primary role is to navigate an autonomous actor through a set of story events, so one way emergent storytelling can be performed is by allowing the autonomous virtual actor to sometimes ignore any manipulation detected in its environment or for the story manager to stop attempting to convince the virtual actor after a few iterations of manipulating the environment. This would cause the actor's decision to fall back on the random nature of autonomy and allow for minor emergence to occur. Also, instead of influencing the actor from story event to story event, the story could consist of only a small number of story events and the story manager will only influence the actor towards a particular story event once the actor has come within some "distance" of the event. For example, a virtual actor could explore their environment, and depending on whether they head east or west, the story manager will guide them to a story event that pertains to the direction they travelled. Also, the "distance" to a story event could be determined by the personality of the actor rather than a physical location. An example of this is if the virtual actor is allowed to emerge over time as being good or evil natured, the story manager can influence the actor towards an appropriate story event for their alignment. Finally, emergent storytelling can be achieved by giving the story manager less of a role in the telling of the story. If the story manager is not attempting to guide the actor down a particular path, the actor is much more free to exercise its autonomy and emerge the story in its own direction. Instead, the story manager could have roles indirectly related to the story. Some examples include manipulating the environment to place unseen boundaries on an actor's range of exploration or enforce a particular genre by creating and maintaining an appropriate environment, 
or even delegating the role of the story manager to the audience to let them personally manipulate the environment in order to emerge the story.

While the audience was not a part of the above demonstration, storytelling with environmental manipulation can be enhanced to allow for the audience to influence the telling of a story. Especially in the case of multiple event-based stories or emergent stories, the story manager can be used to accent the audience's influence or vice versa. Consider an example where the audience's character robs an actor's house when he is gone but is seen by other actors as the character escapes. Without any additional interaction, the robbed actor realizes that it has been robbed, but does not know what it can do about it. However, the story manager could be used to convince the witnesses to talk with the robbed actor about, for example, what the audience's character looked like or put up "wanted" posters throughout town. While the audience manipulated the actor's environment by stealing its possessions, the story manager accents that action by convincing the virtual residents of the town to seek justice by pursuing the audience's character. Likewise, in the previous example about an actor needing to travel through a mountain trail, a forest path, or an open plain, the audience's influence could be used to accent what the story manager has already established. For example, the audience's character could give the actor hiking boots - slightly influencing the actor towards the mountain trail - in order to sway the actor's decision within the choices presented by the story manager.

Because the audience is a part of a manipulatable environment, the story manager can be used to resolve situations in which audience interaction is counter to the story. Especially when performing a story with a single story, the audience's influence could play a negative role in the telling of the story. For example, in a love story between two virtual actors in which the audience's character has a great deal of freedom, the story could be ruined simply by pushing one of the main actors off of a building. Because of this possible negative role, the story manager has several responses it can possibly perform: it can manipulate capabilities of the audience, allow for a premature end to the story, counter the actions that the audience has performed, or temporarily prevent audience interaction. While limiting the audience's capabilities is simple and effective, it may break the audience's suspension of disbelief because they feel their limitations and thus are reinforced that they are in an imaginary world. Oppositely, the audience could be allowed to do whatever they want, and the results of their actions could end the story prematurely. 
For example, Morrowind allows the player to kill any virtual actor through the world, but if an actor vital to the main story is killed, the player is given this message:

With this character's death, the thread of prophecy is severed. Restore a saved game to restore the weave of fate or persist in the doomed world you have created. [Morrowind]

While the player is still allowed to explore the world, the main story cannot be concluded. Additionally, the story manager can also be used to counter the audience's actions through its own environmental manipulation. For example, if the audience's character lies a trap that an important virtual actor will soon walk into, the story manager can convince the actor to take a different path. This method of resolution is the most effective because the story manager does not limit the audience's capabilities, take control of the virtual actor, nor allow for the audience to end the story prematurely, thus maintaining the believability of the situation and the progression of the story; the audience's plans are simply foiled by the story manager. Finally, in the worst case, the story manager can decide to temporarily prohibit audience interaction in some way. While not desirable, this would be less restrictive than with other storytelling approaches and come much later as a last resort because of the ability of the story manager to handle audience interaction by first attempting to counter their actions through environmental manipulation.

\section{CONCLUSION}

Molding an environment around a virtual actor with the help of a story manager, whether physically or just the actor's perception of it, gives the actor the ability to reliably perform stories with event-based stories while preserving the believability and reusability of the actor and environment. While autonomous actors have traditionally been used only for emergent storytelling, this new storytelling method enables them to be used reliably and efficiently to tell event-based stories as well, reaping the benefits of their autonomous nature. In addition, the separation of the virtual actors from the environment and story manager in terms of design promotes a cleaner, reusable architecture that also allows for independent development and improvement. By modeling artificial intelligence design after Herbert Simon's "artifact," emphasizing the encapsulation of the inner mechanisms of virtual actors, the next era of digital storytelling can be driven by the design and development of reusable storytelling components and the interaction 
between the virtual actor, its environment, and the story manager.

The notion of digital free will also opens the door to more personified virtual actors. With the focus shifting from utilitarian virtual actors, designed solely for a single medium, to multi-faceted autonomous actors for adaptable purposes, the gap between human and artificial intelligent thought processes can begin to decrease. Virtual actors can be designed as living, breathing digital creatures with a wide array of personalities so that, like humans, they can adapt to the various environments that they are placed in. Marvin Minsky, the co-founder of the MIT Artificial Intelligence Laboratory, has expressed disappointment that artificial intelligence research has not made advancements towards building fully autonomous thinking machines [Minsky]. Perhaps digital storytelling is the medium for Minsky's vision to reach fruition. 


\section{BIBLIOGRAPHY ${ }^{\dagger}$}

[B\&W] Lionhead Studios (2001). Black \& White. Redwood City, CA: EA Games. http://www.lionhead.com/bw/ (Retrieved February 20, 2010)

[Barnes] Barnes, J. \& Hutchens, J. (2002). AI Game Programming Wisdom: Scripting for Undefined Circumstances. Hingham, MA: Charles River Media.

[Cavazza] Cavazza, M., Charles, F., \& Mead, S. J. (2002) Character-Based Interactive Storytelling. IEEE Intelligent Systems, 17(4), pp. $17-24$

[CYOA] Demian's Gamebook Web Page. Choose Your Own Adventure. Retrieved July 3, 2006 from

http://www.gamebooks.org/show_series.php?name=Choose+Your+Own+Adventure

[Façade] Façade: A One-Act Interactive Drama.

Retrieved July 3, 2006 from http://www.interactivestory.net

[F.E.A.R.] Monolith Productions, Inc. (2005). F.E.A.R. (First Encounter Assault Recon). Vivendi Universal.

http://www.whatisfear.com/ (Retrieved February 20, 2010)

[FFVI] Square Co., Ltd. (1994). Final Fantasy VI. Foster City, CA: Sony.

http://na.square-enix.com/games/ff7/ (Retrieved February 20, 2010)

[Fiddler] Stein, J., Bock, J., \& Harnick., S. (1964). Fiddler on the Roof.

[GTA3] DMA Design (2001). Grand Theft Auto III. New York City, NY: Rockstar Games. http://www.rockstargames.com/grandtheftauto3/ (Retrieved February 20, 2010)

[Guild Wars] Arena.net (2005). Guild Wars. Seattle, WA: NCSoft. http://www.guildwars.com (Retrieved February 20, 2010)

[GWOnline] Guild Wars Unofficial Forums.

Retrieved July 3, 2006 from http://forums.gwonline.net

[Half-Life 2] Valve Corporation (2004). Half-Life 2. Kirkland, WA: Valve Corporation. http://orange.half-life2.com/ (Retrieved February 20, 2010)

[Havok] Havok (2000). Havok Physics.

Retrieved July 3, 2006 from http://www.havok.com

[Link] Nintendo (1992). The Legend of Zelda: A Link to the Past. Redmond, WA: Nintendo. http://www.zelda.com/universe/game/past/ (Retrieved February 20, 2010)

[Magerko] Magerko, B. et al. (2004). AI Characters and Directors for Interactive Computer Games. Retrieved July 3, 2006 from http://ai.eecs.umich.edu/people/laird/papers/magerko-2004-IAAI-Haunt.pdf

[Mateas] Mateas, M. \& Stern, A. (2002). A Behavior Language for Story-Based Believable Agents. IEEE Intelligent Systems, 17(4), pp. $39-47$

$\dagger$ Internet references are cited as "Retrieved [date] from [URL]," but references to video game content are cited as "[URL] (Retrieved [date])" in which the URL provides more information about the video game itself. 
[Minsky] Wired News (2003). AI Founder Blasts Modern Research.

Retrieved July 3, 2006 from http://www.wired.com/news/technology/0,1282,58714,00.html

[Morrowind] Bethesda Game Studios (2002). Elder Scrolls III: Morrowind. Bethesda, MD: Bethesda Softworks / ZeniMax.

http://www.elderscrolls.com/home/home.php (Retrieved February 20, 2010)

[Orkin] Orkin, J. (2004). AI Game Programming Wisdom 2: Constraining Autonomous Character Behavior with Human Concepts. Hingham, MA: Charles River Media.

[Scribblenauts] $5^{\text {th }}$ Cell (2009). Scribblenauts. Burbank, CA: Warner Bros. Interactive Entertainment. http://games.kidswb.com/scribblenauts/ (Retrieved February 20, 2010)

[Simon] Simon, H. (1981). The Sciences of the Artificial. Massachusetts Institute of Technology.

[SMB] Nintendo EAD (1985). Super Mario Bros. Redmond, WA: Nintendo.

http://www.nintendo.com/ (Retrieved February 20, 2010)

[Torchlight] Runic Games, Inc. (2009). Torchlight. Los Angeles, CA: Encore, Inc.

http://www.torchlightgame.com/ (Retrieved February 20, 2010)

[Tozour] Tozour, P. (2002). AI Game Programming Wisdom: The Perils of AI Scripting. Hingham, MA: Charles River Media.

[Warcraft] Blizzard Entertainment, Inc. (2002). Warcraft III: Reign of Chaos. Irvine, CA: Blizzard Entertainment, Inc.

http://us.blizzard.com/en-us/games/war3/ (Retrieved February 20, 2010) 